\section{Agriculture, fisheries and food}

\section{Output and input}

(Tables 21.1 and 21.2)

For both tables, output is net of VAT collected on the sale of non-edible products. Figures for total output include subsidies on products, that is payments that have the purpose of influencing production, their prices or remuneration of the factors of production. Unspecified crops include turf, other minor crops and arable area payments for fodder maize. Eggs include the value of duck eggs and exports of eggs for hatching. Landlords' expenses are included within farm maintenance, miscellaneous expenditure and depreciation of buildings and works. Also included within 'Other farming costs' are livestock and crop costs, water costs, insurance premia, bank charges, professional fees, rates, and other farming costs.

\section{Other subsidies}

These are payments other than subsidies on products from which farmers can benefit as a consequence of engaging in agriculture. Include environment and countryside management schemes, organic farming schemes, support schemes for less favoured areas, Single Payment Scheme, animal disease compensation attributable to income, and other payments.

\section{Compensation of employees and interest charges}

Total compensation of employees excludes the value of work done by farm labour on own account, capital formation in buildings and work. 'Interest' relates to interest charges on loans for current farming purposes and buildings, less interest on money held on short-term deposit.

\section{Rent}

Rent paid (after deductions) is the rent paid on all tenanted land including 'conacre' land in Northern Ireland, less landlords' expenses and the benefit value of dwellings on that land. Rent received (after deductions) is the rent received by farming landowners from renting of land to other farmers, less landlords' expenses and the benefit value of dwellings on that land. Total net rent is the net rent flowing out of the agricultural sector paid to non-farming landowners, including that part of tenanted land in Northern Ireland.

\section{Agricultural censuses and surveys}

(Tables 21.3, 21.5 and 21.13)

The coverage for holdings includes all main and minor holdings for each country. Northern Ireland data are now based on all active farm business.

\section{Estimated quantity of crops and grass harvested}

(Table 21.4)

The estimated yield of sugar beet is obtained from production figures supplied by British Sugar plc in England and Wales. In Great Britain potato yields are estimated in consultation with the Potato Council Limited.

\section{Forestry}

(Table 21.6)

Statistics for state forestry are from Forestry Commission and Forest Service management information systems. For private forestry in Great Britain, statistics on new planting and restocking are based on records of grant aid and estimates of planting undertaken without grant aid, and softwood production is estimated from a survey of the largest timber harvesting companies. Hardwood production is estimated from deliveries of roundwood to primary wood processors and others, based on surveys of the UK timber industry, data provided by trade associations and estimates provided by the Expert Group on Timber and Trade Statistics.

\section{Average weekly earnings and hours of agricultural and horticultural workers}

(Tables 21.11 and 21.12)

Prior to 1998 , data were collected from a monthly postal survey, which mainly covered male full-time workers. Between 1998 and 2002 the survey collected information on an annual basis via a telephone survey. The survey was reviewed in 2002 and it was concluded that the frequency of the survey should be increased to four times per year to enable the production of more representative annual estimates. The annual sample size has been retained and has been split between four quarterly telephone surveys.

From April 2009, publication of quarterly results ceased. Results are now published on an annual basis, for the 12 months ending in September. The annual data provides the more robust estimates of earnings and hours, however quarterly data collection continues in order to achieve a representative sample of workers across the year. 
The survey covers seven main categories of workers, providing data which are used by the Agricultural Wages Board when considering wage claims and in considering the cost of labour in agriculture and horticulture.

Data on earnings represents the total earnings for workers aged 20 and over. Figures include all payments-in-kind, valued where applicable in accordance with the Agricultural Wages Order. Part-time workers are defined as those working less than 39 basic hours per week. Casual workers are those employed on a temporary basis.

Results can be found on the Department for Environment, Food and Rural Affairs (DEFRA) website at: www.defra.gov.uk

\section{Fisheries}

(Table 21.15)

Figures show the number of registered and licensed fishing vessels, based on information provided by the Registry of Shipping and Seamen and licence registers maintained by the Marine and Fisheries Agency for England and Wales, the Scottish Government and the Department of Agriculture and Rural Development in Northern Ireland.

\section{Estimated average household food consumption - 'Family Food' Expenditure and Food Survey}

\section{(Table 21.16)}

In 2008 the Expenditure and Food Survey (EFS) was renamed as the Living Costs and Food Survey (LCFS) when it became part of the Integrated Household Survey (IHS). The Expenditure and Food Survey started in April 2001, having been preceded by the National Food Survey (NFS) and the Family Expenditure Survey (FES). Both surveys were brought into one to provide value for money without compromising data quality. The EFS was effectively a continuation of the FES, extended to record quantities of purchases. This extension is now known as the Family Food Module of the LCFS. Estimates from the NFS prior to 2000 have been adjusted by aligning estimates for the year 2000 with corresponding estimates from the FES. From 2006 the survey moved onto a calendar year basis (from the previous financial year basis) in preparation for its integration to the Integrated Household Survey from January 2008.

The Living Costs and Food Survey is a voluntary sample survey of private households throughout the UK. The basic unit of the survey is the household, which is defined as a group of people living at the same address and sharing common catering arrangements. The survey is continuous, interviews being spread evenly over the year to ensure that seasonal effects are covered. Each household member over the age of seven keeps a diary of all their expenditure over a two-week period. A simplified version of the diary is used by those aged 7 to 15. The diaries record expenditure and quantities of purchases of food and drink rather than consumption of food and drink. Items of food and drink are defined as either household or eating out and are recorded in the form in which the item was purchased not how it was consumed. 'Household' covers all food that is brought into the household. 'Eating out' covers all food that never enters the household, for example restaurant meals, school meals and snacks eaten away from home.

In 2008 the Living Costs and Food Survey collected the diaries of 13,890 people within 5,845 households across the UK. The response rate for 2008 was 51 per cent in Great Britain and 54 per cent in Northern Ireland. 


\section{Production and income account at current prices ${ }^{1}$

\begin{tabular}{|c|c|c|c|c|c|c|c|c|c|c|c|c|}
\hline & & 1999 & 2000 & 2001 & 2002 & 2003 & 2004 & 2005 & 2006 & 2007 & 2008 & 2009 \\
\hline \multicolumn{13}{|l|}{ Output $^{3}$} \\
\hline 1.Total cereals: & C5x5 & 1620.2 & 1621.4 & 1348.3 & 1534.7 & 1491.3 & 1707.5 & 1434.9 & 1506.1 & 1949.5 & 3152.8 & 2352.8 \\
\hline Wheat & KFKA & 1105.2 & 1124.8 & 836.6 & 1095.1 & 1000.8 & 1232.3 & 1018.3 & 1066.4 & 1324.6 & 2245.0 & 1589.6 \\
\hline Rye & VQBG & 1.8 & 22.0 & 5.6 & 1.2 & 1.4 & 1.6 & 1.3 & 1.4 & 2.3 & 1.9 & 1.8 \\
\hline Barley & KFKB & 474.6 & 432.1 & 465.7 & 392.0 & 445.7 & 433.1 & 379.5 & 383.8 & 555.4 & 817.4 & 686.5 \\
\hline Oats and summ & KFKC & 37.9 & 41.7 & 39.8 & 45.5 & 42.5 & 39.7 & 34.8 & 53.6 & 66.2 & 87.1 & 72.9 \\
\hline Other cereals & VQBH & 0.8 & 0.8 & 0.6 & 0.9 & 0.9 & 0.9 & 0.9 & 0.9 & 1.1 & 1.4 & 1.9 \\
\hline 2. Total industrial crops & VQBI & 783.3 & 699.4 & 773.8 & 859.8 & 812.3 & 798.5 & 814.1 & 731.6 & 769.0 & 1164.5 & 1108.1 \\
\hline Oilseeds & VQBJ & 225.0 & 143.8 & 177.1 & 220.1 & 313.7 & 265.9 & 277.2 & 315.1 & 426.6 & 628.2 & 492.7 \\
\hline Oilseed rape & KFKG & 194.8 & 139.0 & 171.5 & 217.4 & 303.8 & 256.7 & 261.2 & 307.2 & 422.3 & 617.8 & 475.4 \\
\hline Other oil seeds & KIBT & 30.1 & 4.8 & 5.6 & 2.7 & 9.9 & 9.2 & 15.9 & 7.9 & 4.3 & 10.4 & 17.4 \\
\hline Sugar beet & KFKH & 279.7 & 252.1 & 256.4 & 282.9 & 279.7 & 278.1 & 279.0 & 178.2 & 161.5 & 208.3 & 240.7 \\
\hline Other industrial crops & VQBK & 278.7 & 303.4 & 340.3 & 356.8 & 218.9 & 254.4 & 257.9 & 238.3 & 180.9 & 328.0 & 374.6 \\
\hline Fibre plants & VQBL & 2.3 & 1.3 & 1.7 & 1.0 & 1.8 & 1.2 & 0.9 & 0.9 & 0.4 & 1.0 & 0.2 \\
\hline Hops & KFKI & 12.1 & 10.4 & 9.0 & 7.2 & 6.1 & 5.7 & 5.4 & 4.4 & 4.4 & 4.4 & 4.4 \\
\hline Others ${ }^{4}$ & VQBM & 264.2 & 291.7 & 329.6 & 348.5 & 211.1 & 247.5 & 251.7 & 233.0 & 176.0 & 322.6 & 370.0 \\
\hline 3.Total forage plants & VQBO & 75.9 & 80.9 & 103.4 & 90.3 & 103.7 & 93.3 & 94.9 & 85.9 & 106.3 & 109.9 & 127.7 \\
\hline \multicolumn{13}{|l|}{ 4. Total vegetables and horticultural } \\
\hline products & VQBP & 1667.5 & 1561.1 & 1612.6 & 1591.1 & 1672.6 & 1621.5 & 1690.0 & 1748.1 & 1848.0 & 1903.2 & 1932.5 \\
\hline 5. Total potatoes (including seeds) & KFKO & 769.4 & 477.1 & 701.4 & 505.0 & 548.3 & 695.2 & 531.1 & 638.4 & 684.4 & 767.2 & 644.3 \\
\hline 6. Total fruit & KFKQ & 256.9 & 232.2 & 238.8 & 251.2 & 310.3 & 315.8 & 388.1 & 382.8 & 467.4 & 544.5 & 570.6 \\
\hline 7.Other crop products including seeds & VQBQ & 42.0 & 37.7 & 37.5 & 25.5 & 31.6 & 31.0 & 52.0 & 47.9 & 43.2 & 44.6 & 45.8 \\
\hline 8. Total crop output (Sum 1 to 7 ) & VQBR & 5215.2 & 4709.7 & 4815.8 & 4857.6 & 4970.0 & 5262.7 & 5004.9 & 5140.8 & 5867.7 & 7686.6 & 6781.8 \\
\hline 9.Total livestock production & VQBS & 4326.7 & 4358.0 & 4277.9 & 4573.0 & 4813.5 & 4813.9 & 4908.4 & 5093.8 & 5230.7 & 6576.4 & 740.1 \\
\hline Primarily & KFLA & 3929.5 & 3964.2 & 3651.3 & 3866.4 & 4083.5 & 4156.5 & 4298.9 & 4362.1 & 4431.2 & 5499.6 & 5928.8 \\
\hline Cattle & KFKU & 1145.2 & 1093.7 & 955.3 & 1145.8 & 1227.1 & 1279.2 & 1465.2 & 1559.2 & 1621.9 & 2068.0 & 2199.6 \\
\hline Pigs & KFKW & 784.8 & 800.2 & 748.4 & 687.2 & 671.1 & 680.0 & 676.9 & 685.2 & 736.3 & 865.2 & 1015.0 \\
\hline Sheep & VQBT & 574.8 & 616.8 & 438.0 & 613.4 & 696.4 & 725.7 & 685.9 & 709.0 & 640.6 & 797.8 & 962.1 \\
\hline Poultry & KFXX & 1275.8 & 1300.9 & 1354.6 & 1262.4 & 1327.7 & 1305.9 & 1300.0 & 1233.1 & 1249.0 & 1578.4 & 1562.9 \\
\hline Other animals & KFKY & 148.9 & 152.6 & 154.9 & 157.7 & 161.2 & 165.7 & 170.9 & 175.5 & 183.5 & 190.3 & 189.2 \\
\hline Gross fixe & KFLI & 397.3 & 393.8 & 626.6 & 706.7 & 730.0 & 657.3 & 609.4 & 731.8 & 799.5 & 1076.8 & 1211.4 \\
\hline Cattle & KUJ & 206.9 & 192.9 & 371.3 & 392.2 & 447.7 & 337.4 & 360.9 & 447.3 & 492.9 & 784.5 & 813.1 \\
\hline Pigs & LUKB & 6.9 & 5.6 & 5.3 & 7.4 & 7.0 & 7.6 & 6.2 & 8.0 & 5.3 & 6.4 & 9.1 \\
\hline Sheep & LUKA & 56.7 & 63.9 & 122.5 & 177.5 & 145.8 & 176.4 & 111.5 & 146.0 & 152.6 & 123.7 & 200.6 \\
\hline Poultry & LUKC & 126.8 & 131.4 & 127.5 & 129.5 & 129.5 & 135.9 & 130.8 & 130.5 & 148.7 & 162.2 & 188.5 \\
\hline 10. Total liv & KFLF & 2963.1 & 2711.4 & 3088.2 & 2834.3 & 3030.7 & 3037.7 & 009.5 & 2918.1 & 3285.9 & 4018.6 & 3692.9 \\
\hline Milk & KFLB & 2662.0 & 2385.8 & 2742.6 & 2466.3 & 2628.5 & 2610.4 & 2592.5 & 2497.2 & 2823.4 & 3446.8 & 3114.0 \\
\hline $\mathrm{Eg}$ & KFLC & 254.0 & 280.3 & 307.1 & 314.5 & 336.4 & 378.3 & 349.4 & 361.7 & 410.1 & 520.2 & 526.2 \\
\hline & $\mathrm{KFL}$ & 21.4 & 22.7 & 17.2 & 19.1 & 20.8 & 20.1 & 19.5 & 11.5 & 11.6 & 10.4 & 9.9 \\
\hline Other animal products & KFLE & 25.7 & 22.6 & 21.2 & 34.5 & 45.1 & 28.9 & 48.1 & 47.7 & 40.8 & 41.2 & 42.9 \\
\hline 11. Total livestock output $(9+10)$ & VQBV & 7289.8 & 7069.4 & 7366.0 & 7407.4 & 7844.2 & 7851.5 & 7917.8 & 8012.0 & 8516.7 & 10595.0 & 10833.1 \\
\hline 12. Total & LUOS & 457188 & 455401 & 473519 & 508574 & 459754 & 462633 & 481053 & 522722 & 474287 & 514235 & 844 \\
\hline $\mathrm{Ag}$ & LUK & & 587.0 & 604.0 & 601.4 & 592.3 & 636.2 & 630.7 & 622.6 & 679.9 & 794.3 & 843.8 \\
\hline Leasing out quote & VQBW & 116.5 & 51.2 & 28.1 & 42.8 & 40.2 & 82.0 & 8.6 & 0.9 & 0.4 & 0.4 & 0.2 \\
\hline \multicolumn{13}{|l|}{ 13. Total inseparable no } \\
\hline activities & LUOT & 273476 & 370184 & 367736 & 450749 & 480252 & 573382 & 712489 & 810993 & 889723 & 1136074 & 809 \\
\hline $\begin{array}{l}\text { 14.Gross output at market prices } \\
(8+11+12+13)\end{array}$ & LUOV & 23140 & 20367 & 17682 & 20364 & 59534 & 71485 & 68120 & 147106 & 71386 & 19425 & 19268 \\
\hline \multirow{2}{*}{\multicolumn{13}{|c|}{ 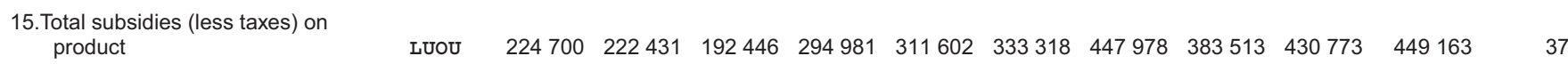 }} \\
\hline & LUOU & 224700 & 222431 & 192446 & 294981 & 311602 & 333318 & 447978 & 383513 & 430773 & 449163 & 37 \\
\hline 16. Output at basic prices $(14+15)$ & KFLT & 15875.0 & 14917.1 & 15187.8 & 15410.9 & 16015.1 & 16637.4 & 14452.4 & 14587.0 & 15894.8 & 19946.2 & 19304.7 \\
\hline & & & & & & & & & & & & \\
\hline Feed wheat & LUNQ & 64.4 & 40.1 & 41.1 & 41.9 & 70.1 & 103.8 & 85.9 & 83.4 & 100.0 & 138.2 & 125.5 \\
\hline $\mathrm{F}$ & LUN & 147.9 & 137.8 & 148.9 & 144.6 & 149.2 & 148.6 & 136.1 & 142.0 & 177.9 & 207.7 & 157.3 \\
\hline & $\mathrm{LU}$ & 14.5 & 12.6 & 12.5 & 11.7 & 11.8 & 13.5 & 11.9 & 15.3 & 19.3 & 23.5 & 19.3 \\
\hline Seed potatoes & LUNT & 29.0 & 6.9 & 13.8 & 12.0 & 4.1 & 9.2 & 12.5 & 15.9 & 8.5 & 12.6 & 10.6 \\
\hline Str & $\mathrm{LU}$ & 232.9 & 258.6 & 291.2 & 306.5 & 177.0 & 209.0 & 210.4 & 191.0 & 137.4 & 266.5 & 307.8 \\
\hline Cor & LUNV & 609.6 & 587.0 & 604.0 & 601.4 & 592.3 & 636.2 & 630.7 & 622.6 & 679.9 & 794.3 & 843.8 \\
\hline Leasir & LUNW & 116.5 & 51.2 & 28.1 & 42.8 & 40.2 & 82.0 & 8.6 & 0.9 & 0.4 & 0.4 & 0.2 \\
\hline Total capital formation in livestock & LUNX & 397.3 & 393.8 & 626.6 & 706.7 & 730.0 & 657.3 & 609.4 & 731.8 & 799.5 & 1076.8 & 1211.4 \\
\hline
\end{tabular}




\section{Production and income account at current prices ${ }^{1}$

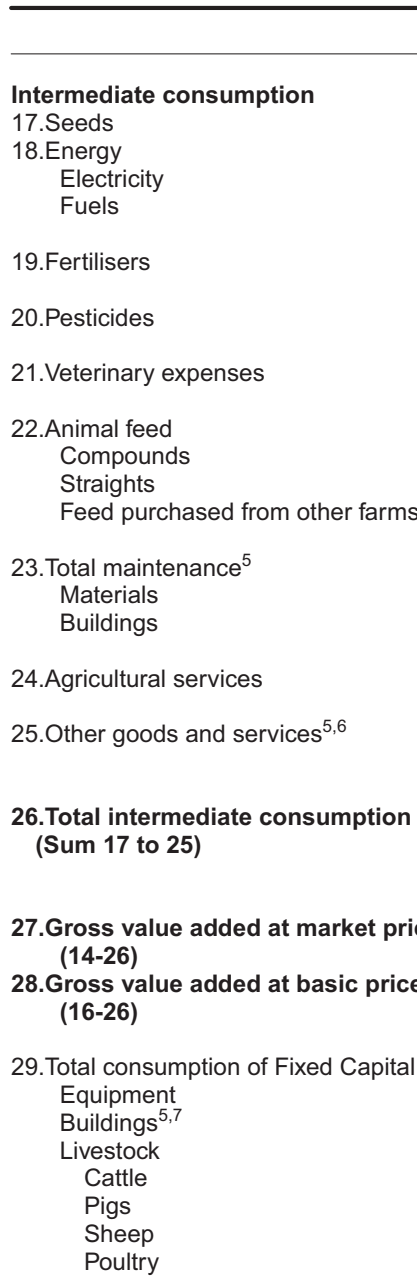

30.Net value added at market prices (27-29)

31.Net value added at basic prices (28-29)

32.Compensation of employees ${ }^{8}$

33. Other taxes on production

34.Other subsidies on production Animal disease compensation Set-aside

Agri-environment schemes ${ }^{9}$

LUOG $\quad 4700.4 \quad 4391.7 \quad 4694.3 \quad 4914.2 \quad 5444.8 \quad 4976.1 \quad 4912.1 \quad 5135.4 \quad 5617.0 \quad 7600.1 \quad 7130.8$

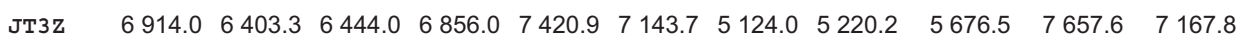

$\begin{array}{llllllllllll}\text { KCPS } & 2438.2 & 2495.6 & 2600.2 & 2584.2 & 2648.2 & 2533.0 & 2659.5 & 2677.4 & 2710.8 & 3060.8 & 3285.3\end{array}$

$\begin{array}{llllllllllllll}\text { KCPR } & 1317.6 & 1267.4 & 1262.9 & 1261.9 & 1205.7 & 1192.4 & 1204.5 & 1194.5 & 1205.6 & 1259.8 & 1363.2\end{array}$

$\begin{array}{llllllllllll}\text { LUOH } & 701.3 & 691.2 & 686.4 & 689.5 & 692.3 & 673.9 & 675.7 & 685.3 & 696.4 & 707.5 & 725.2\end{array}$

$\begin{array}{llllllllllll}\text { VQEA } & 419.3 & 537.0 & 650.8 & 632.9 & 750.1 & 666.7 & 779.3 & 797.6 & 808.7 & 1093.5 & 1196.9\end{array}$

$\begin{array}{llllllllllll}\text { LUOI } & 208.2 & 281.1 & 348.4 & 353.2 & 441.2 & 363.6 & 489.6 & 499.4 & 503.3 & 745.5 & 755.6\end{array}$

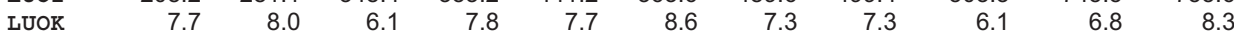

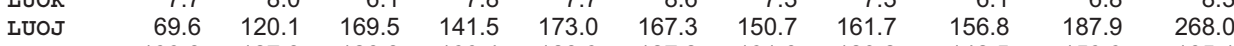

$\begin{array}{llllllllllll}\text { LUOL } & 133.8 & 127.8 & 126.8 & 130.4 & 128.3 & 127.2 & 131.6 & 129.2 & 142.5 & 153.3 & 165.1\end{array}$

$\begin{array}{llllllllllll}\text { KCPT } \quad 2262.2 & 1896.1 & 2 & 2894.2 & 2329.9 & 2796.6 & 2443.1 & 2252.6 & 2458.0 & 2906.2 & 4539.3 & 3845.5\end{array}$

35. Net value added at factor cost

JT42

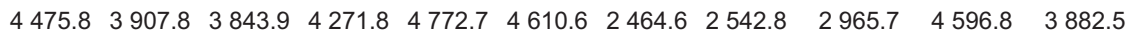

LUOR

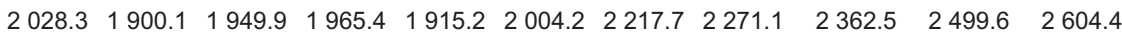

36. Rent$$
\text { incer }
$$

$\begin{array}{ll}\text { Rent } & \text { KCPV } \\ \text { Paid }^{11} & \text { ZBXE } \\ \text { Received }^{12} & \text { ZBXF }\end{array}$

37. Interest ${ }^{13}$

Total income from farming (35-32-36-37)

ZBXE

$\begin{array}{rrrrrrrrrrr}-92.4 & -92.2 & -84.9 & -80.6 & -82.8 & -95.7 & -102.2 & -98.9 & -102.0 & -105.6 & -104.2 \\ & & & & & & & & & & \\ 470.1 & 462.1 & 695.7 & 723.4 & 782.1 & 777.7 & 2818.4 & 2943.5 & 2955.7 & 3235.8 & 3606.6 \\ 11.9 & 19.1 & 13.0 & 24.6 & 24.7 & 18.6 & 19.9 & 16.9 & 21.2 & 25.6 & 21.7 \\ 170.0 & 127.3 & 180.1 & 142.5 & 176.7 & 129.5 & - & . . & . . & . . & . . \\ 128.5 & 140.3 & 164.1 & 196.1 & 222.6 & 257.0 & 287.6 & 376.1 & 460.0 & 490.3 & 496.8 \\ 159.6 & 175.4 & 338.5 & 360.2 & 358.1 & 372.6 & 2510.9 & 2550.5 & 2474.4 & 2719.9 & 3088.1 \\ 4853.5 & 4277.7 & 4454.7 & 4914.6 & 5472.0 & 5292.6 & 5180.8 & 5387.4 & 5819.4 & 7727.0 & 7384.8\end{array}$

$\begin{array}{lllllllllll}239.6 & 224.5 & 250.5 & 253.7 & 268.6 & 241.2 & 219.7 & 237.0 & 254.9 & 273.6 & 267.7\end{array}$ $\begin{array}{lllllllllll}239.6 & 224.5 & 250.5 & 253.7 & 268.6 & 241.2 & 219.7 & 237.0 & 254.9 & 273.6 & 267.7 \\ 322.0 & 303.3 & 328.5 & 339.6 & 364.7 & 346.5 & 304.7 & 325.6 & 352.3 & 368.8 & 367.1\end{array}$ $\begin{array}{lllllllllll}-82.4 & -78.8 & -78.1 & -85.9 & -96.2 & -105.2 & -85.0 & -88.6 & -97.3 & -95.2 & -99.4\end{array}$

1 See chapter text.

$\begin{array}{lc}1 \text { See chapter text. } & 9 \text { Includes environment and countryside management schemes and Organic } \\ 2 \text { Provisional. } & \text { Farming Schemes. }\end{array}$

$\begin{array}{llllllllllll}\mathrm{KCPU} & 68.6 & 83.9 & 101.6 & 118.5 & 134.9 & 152.6 & 174.2 & 200.2 & 224.8 & 252.4 & 267.0\end{array}$ $\begin{array}{lllllllllllll}\text { KCQB } & 1997.6 & 1540.9 & 1711.7 & 2235.3 & 2854.9 & 2550.1 & 2210.7 & 2343.7 & 2568.2 & 4361.7 & 4069.1\end{array}$

3 Output is net of VAT collected on the sale of non-edible products. Figures 10 Includes Single Payment Scheme, support for less favoured areas and other for total output include subsidies on products, but not other subsidies.

4 Includes straw and minor crops. payments received by farmers by virtue of engaging in agriculture. 4 Includes straw and minor crops.
5 Landlords' expenses are included within 'Total maintenance', 'Other goods 11 Rent paid on all tenanted land (including 'conacre' land in Northern Ireland) and services' and 'Total consumption of Fixed Capital of buildings'. fit value of dwellings on that land.

6 Includes livestock and crop costs, water costs, insurance premiums, bank 12 Rent received by farming landowners from renting of land to other farmers less charges, professional fees, rates and other farming costs. landlords' expenses. This series starts in 1996 following a revision to the

7 A more empirically based methodology for calculating landlords' consumpmethodology of calculating net rent.

tion of fixed capital was introduced in 2000. The new series has been linked 13 Interest charges on loans for current farming purposes and buildings and with the old one using a smoothing procedure for the transition year of works less interest on money held on short term deposit. 1996.

8 Excludes the value of work done by farm labour on own account capital forSource: Department for Environment, Food and Rural Affairs: 01904455080 mation in buildings and works. 


\section{Output and input volume indices ${ }^{1}$

Indices $(2000=100)$

\begin{tabular}{|c|c|c|c|c|c|c|c|c|c|c|c|c|}
\hline & & 1999 & 2000 & 2001 & 2002 & 2003 & 2004 & 2005 & 2006 & 2007 & 2008 & 2009 \\
\hline \multicolumn{13}{|l|}{ Outputs } \\
\hline 1. Total cereals: & VQAN & 92.5 & 100.0 & 80.4 & 97.4 & 91.6 & 93.5 & 89.5 & 88.6 & 81.5 & 102.6 & 93.7 \\
\hline Wheat & LUKH & 88.9 & 100.0 & 70.1 & 96.0 & 86.5 & 93.2 & 89.7 & 88.7 & 79.6 & 103.0 & 87.0 \\
\hline Rye & VQAO & 104.5 & 100.0 & 104.5 & 90.9 & 86.4 & 86.4 & 86.4 & 86.4 & 86.4 & 86.4 & 86.4 \\
\hline Barley & LUKI & 102.4 & 100.0 & 105.3 & 97.2 & 101.4 & 91.8 & 87.5 & 83.7 & 81.5 & 97.2 & 107.2 \\
\hline Oats and summer cereal mixtures & LUKJ & 84.1 & 100.0 & 96.9 & 118.0 & 116.8 & 97.5 & 83.1 & 114.4 & 111.7 & 122.7 & 118.4 \\
\hline Other cereals & VQAP & 82.4 & 100.0 & 74.4 & 108.9 & 107.0 & 102.9 & 104.2 & 104.1 & 87.2 & 99.5 & 154.7 \\
\hline Oil seeds & VQAR & 167.0 & 100.0 & 100.3 & 124.0 & 153.1 & 139.8 & 167.2 & 161.5 & 178.9 & 183.8 & 176.3 \\
\hline Oilseed rape & VQAS & 148.8 & 100.0 & 100.7 & 127.2 & 153.8 & 140.4 & 165.5 & 163.1 & 183.8 & 188.0 & 177.1 \\
\hline Other oil seeds & LUKN & 708.3 & 100.0 & 89.0 & 39.0 & 130.4 & 120.2 & 195.9 & 116.5 & 46.0 & 69.4 & 131.7 \\
\hline Sugar beet & C5 54 & 116.6 & 100.0 & 91.8 & 105.3 & 101.0 & 99.6 & 95.7 & 81.5 & 74.2 & 84.2 & 91.7 \\
\hline Other industrial crops & VQAU & 95.2 & 100.0 & 91.2 & 97.3 & 92.0 & 93.1 & 75.1 & 72.4 & 45.8 & 76.1 & 79.2 \\
\hline Fibre plants & VQAV & 139.6 & 100.0 & 69.3 & 40.3 & 67.3 & 45.5 & 31.7 & 32.5 & 16.1 & 26.8 & 6.7 \\
\hline Hops & LUKP & 112.4 & 100.0 & 94.3 & 94.5 & 72.4 & 72.4 & 61.9 & 51.1 & 51.1 & 51.1 & 51.1 \\
\hline Others $^{3}$ & VQAW & 94.4 & 100.0 & 91.2 & 97.7 & 92.6 & 94.0 & 75.7 & 73.3 & 45.9 & 77.0 & 80.4 \\
\hline \multicolumn{13}{|l|}{ 4. Total vegetables and horticultural } \\
\hline Products: & VQAY & 102.4 & 100.0 & 96.8 & 96.1 & 93.5 & 95.3 & 95.4 & 89.7 & 89.4 & 91.5 & 89.8 \\
\hline Fresh vegetables & LUKX & 106.2 & 100.0 & 96.6 & 88.3 & 87.8 & 88.3 & 91.9 & 89.8 & 86.5 & 89.0 & 89.8 \\
\hline Plants and flowers & LUKZ & 97.5 & 100.0 & 97.0 & 106.6 & 101.3 & 104.8 & 100.4 & 90.0 & 93.8 & 95.3 & 90.4 \\
\hline 5.Total potatoes (including seeds) & LUKW & 120.4 & 100.0 & 113.0 & 110.4 & 100.4 & 105.7 & 98.5 & 93.3 & 89.4 & 95.5 & 96.5 \\
\hline 6. Total fruit & LUKY & 108.2 & 100.0 & 105.8 & 99.8 & 109.7 & 130.0 & 148.5 & 149.7 & 176.5 & 182.8 & 193.2 \\
\hline 7.Other crop products including seeds & VQAZ & 101.8 & 100.0 & 101.2 & 69.0 & 83.0 & 84.6 & 123.6 & 111.5 & 100.7 & 106.5 & 92.9 \\
\hline 8.Total crop output & VQBA & 103.3 & 100.0 & 93.3 & 99.9 & 97.3 & 99.5 & 98.3 & 94.1 & 90.4 & 102.9 & 100.0 \\
\hline 9. Total livestock production & VQBB & 105.3 & 100.0 & 93.6 & 97.2 & 94.7 & 95.4 & 97.7 & 96.1 & 97.2 & 97.9 & 94.7 \\
\hline Mainly for meat processing & LÜLH & 104.7 & 100.0 & 92.1 & 96.2 & 94.9 & 95.4 & 99.3 & 96.9 & 97.2 & 96.5 & 94.9 \\
\hline Cattle & LULC & 103.1 & 100.0 & 88.6 & 102.7 & 105.9 & 104.5 & 118.9 & 114.0 & 116.7 & 114.7 & 113.8 \\
\hline Pigs & LULE & 117.5 & 100.0 & 90.9 & 86.8 & 76.8 & 77.5 & 77.0 & 76.9 & 80.9 & 80.0 & 81.0 \\
\hline Sheep & LULD & 104.1 & 100.0 & 71.8 & 81.8 & 83.3 & 88.6 & 89.9 & 89.7 & 89.3 & 86.8 & 83.3 \\
\hline Poultry & LULF & 99.5 & 100.0 & 104.6 & 102.3 & 101.0 & 100.2 & 99.1 & 95.9 & 91.6 & 93.4 & 90.3 \\
\hline Pigs & LULP & 148.1 & 100.0 & 85.2 & 126.6 & 101.2 & 93.5 & 79.5 & 96.9 & 88.6 & 99.7 & 99.3 \\
\hline Sheep & LULO & 147.5 & 100.0 & 155.6 & 173.6 & 119.4 & 148.3 & 116.3 & 138.4 & 153.3 & 106.7 & 113.6 \\
\hline Poultry & LULQ & 96.7 & 100.0 & 67.8 & 68.9 & 68.9 & 72.3 & 69.6 & 69.4 & 79.1 & 86.3 & 100.3 \\
\hline 10. Total livestock products & LULM & 102.8 & 100.0 & 101.6 & 102.9 & 104.0 & 101.6 & 101.8 & 100.6 & 98.3 & 96.9 & 96.2 \\
\hline Milk & LULI & 102.8 & 100.0 & 101.2 & 102.3 & 103.5 & 100.3 & 99.6 & 98.7 & 96.8 & 94.6 & 93.7 \\
\hline Eggs & LULU & 101.1 & 100.0 & 107.3 & 107.1 & 104.9 & 113.3 & 115.2 & 111.3 & 108.6 & 115.5 & 116.1 \\
\hline Raw wool & LULK & 103.9 & 100.0 & 83.1 & 86.3 & 84.4 & 85.7 & 87.9 & 79.5 & 73.4 & 70.6 & 67.6 \\
\hline Other animal products & LULL & 118.7 & 100.0 & 88.2 & 139.6 & 173.9 & 105.3 & 173.7 & 173.9 & 137.8 & 112.9 & 119.4 \\
\hline 11.Total livestock output & $\mathrm{VQBC}$ & 104.3 & 100.0 & 96.7 & 99.4 & 98.3 & 97.9 & 99.4 & 97.9 & 97.7 & 97.6 & 95.3 \\
\hline 12. Total other agricultural activities & VQBD & 116.2 & 100.0 & 98.9 & 100.7 & 98.7 & 109.8 & 96.2 & 92.0 & 98.4 & 112.7 & 117.3 \\
\hline Agricultural services & VQBE & 105.9 & 100.0 & 102.9 & 102.5 & 100.9 & 106.3 & 103.3 & 100.0 & 107.0 & 122.6 & 127.6 \\
\hline Leasing out quota & VQBF & 234.4 & 100.0 & 54.0 & 80.9 & 73.8 & 146.3 & 15.9 & 1.6 & 0.7 & 0.7 & 0.3 \\
\hline $\begin{array}{l}\text { 13. Total inseparable non-agricultural } \\
\text { Activities }\end{array}$ & LULX & 91.1 & 100.0 & 124.5 & 108.2 & 110.6 & 113.7 & 116.1 & 118.7 & 119.4 & 119.1 & 118.7 \\
\hline
\end{tabular}


Indices $(2000=100)$

\begin{tabular}{|c|c|c|c|c|c|c|c|c|c|c|c|c|}
\hline & & 1999 & 2000 & 2001 & 2002 & 2003 & 2004 & 2005 & 2006 & 2007 & 2008 & $2009^{2}$ \\
\hline 14.Gross output at market prices & VQEG & 104.0 & 100.0 & 96.6 & 100.0 & 98.4 & 99.6 & 99.5 & 97.1 & 95.9 & 101.2 & 98.9 \\
\hline 15. Total subsidies (less taxes) on product & VQEE & 108.2 & 100.0 & 86.0 & 98.8 & 99.6 & 99.6 & 108.5 & 99.9 & 94.5 & 89.3 & 98.3 \\
\hline $\begin{array}{l}\text { 16.Output at basic prices } \\
\text { of which transactions within the } \\
\text { agricultural industry }\end{array}$ & LULY & 104.6 & 100.0 & 95.2 & 99.8 & 98.5 & 99.5 & 100.1 & 97.6 & 96.4 & 101.7 & 99.5 \\
\hline Feed wheat & LULZ & 145.3 & 100.0 & 95.6 & 102.9 & 162.4 & 220.2 & 215.6 & 194.4 & 168.8 & 180.3 & 195.3 \\
\hline Feed oats & LUMB & 109.4 & 100.0 & 104.1 & 102.5 & 110.5 & 110.3 & 97.1 & 110.9 & 111.2 & 106.2 & 107.6 \\
\hline Seed potatoes & LUMC & 141.1 & 100.0 & 109.9 & 109.7 & 43.5 & 65.8 & 131.2 & 138.2 & 53.1 & 76.5 & 75.1 \\
\hline Straw & LUMD & 93.9 & 100.0 & 89.8 & 95.8 & 89.9 & 90.9 & 71.2 & 68.0 & 39.8 & 70.1 & 73.2 \\
\hline Contract work & LUME & 105.9 & 100.0 & 102.9 & 102.5 & 100.9 & 106.3 & 103.3 & 100.0 & 107.0 & 122.6 & 127.6 \\
\hline Leasing of quota & LUMF & 234.4 & 100.0 & 54.0 & 80.9 & 73.8 & 146.3 & 15.9 & 1.6 & 0.7 & 0.7 & 0.3 \\
\hline Total capital formation in livestock & LUMG & 112.3 & 100.0 & 104.9 & 105.4 & 95.9 & 97.8 & 89.6 & 93.4 & 99.0 & 107.1 & 95.4 \\
\hline \multicolumn{13}{|l|}{ Intermediate Consumption } \\
\hline 17.Seeds & LUMO & 111.9 & 100.0 & 99.2 & 84.7 & 82.1 & 75.9 & 92.6 & 100.0 & 112.7 & 108.0 & 106.8 \\
\hline 18.Energy & VQEH & 109.4 & 100.0 & 101.3 & 100.6 & 85.5 & 88.2 & 81.9 & 78.1 & 80.4 & 77.3 & 80.2 \\
\hline Electricity & VQEI & 108.1 & 100.0 & 108.2 & 110.3 & 89.6 & 86.6 & 81.7 & 75.9 & 71.5 & 77.8 & 77.2 \\
\hline Fuels & VQEJ & 110.1 & 100.0 & 97.8 & 95.8 & 83.5 & 88.9 & 81.9 & 79.0 & 84.6 & 77.2 & 81.7 \\
\hline 19.Fertilisers & VQEK & 114.2 & 100.0 & 87.4 & 91.2 & 78.2 & 79.8 & 71.4 & 67.6 & 63.7 & 62.0 & 60.1 \\
\hline 20.Pesticides & LUMQ & 101.4 & 100.0 & 93.9 & 95.8 & 90.5 & 99.3 & 92.3 & 86.4 & 93.8 & 111.1 & 109.2 \\
\hline 21.Veterinary expenses & LUMWW & 104.3 & 100.0 & 95.6 & 100.0 & 97.6 & 104.4 & 105.6 & 100.2 & 105.3 & 123.4 & 124.5 \\
\hline 22.Animal feed & LUML & 102.9 & 100.0 & 104.4 & 101.9 & 105.8 & 107.7 & 105.6 & 105.4 & 103.8 & 105.4 & 105.3 \\
\hline Compounds & LUMH & 108.8 & 100.0 & 102.9 & 102.3 & 102.2 & 103.3 & 99.5 & 104.4 & 107.6 & 106.9 & 105.0 \\
\hline Straights & LUMI & 89.9 & 100.0 & 107.1 & 98.8 & 109.0 & 111.2 & 111.5 & 103.3 & 96.3 & 103.1 & 104.5 \\
\hline Feed purchased from other farms & LUMU & 110.7 & 100.0 & 104.7 & 111.0 & 119.9 & 125.2 & 125.7 & 120.5 & 106.5 & 104.6 & 110.8 \\
\hline 23. Total maintenance ${ }^{4}$ & VQEL & 110.6 & 100.0 & 102.2 & 96.2 & 92.6 & 92.9 & 86.1 & 83.4 & 86.0 & 86.4 & 92.0 \\
\hline Materials & LUMU & 109.5 & 100.0 & 98.5 & 91.0 & 86.3 & 85.4 & 78.7 & 75.2 & 77.7 & 77.6 & 81.6 \\
\hline Buildings & LUMT & 113.1 & 100.0 & 110.5 & 108.1 & 107.1 & 110.2 & 103.4 & 102.9 & 105.6 & 107.0 & 116.9 \\
\hline 25.Other goods and services ${ }^{4,5}$ & VQEO & 112.2 & 100.0 & 93.6 & 93.7 & 99.2 & 105.4 & 99.0 & 94.9 & 92.7 & 96.1 & 98.3 \\
\hline 26. Total intermediate consumption & LUNE & 108.1 & 100.0 & 98.4 & 96.7 & 95.6 & 98.5 & 95.0 & 92.7 & 93.5 & 96.1 & 97.3 \\
\hline 27.Gross value added at market prices & LUNF & 96.6 & 100.0 & 93.3 & 105.9 & 103.6 & 101.6 & 108.4 & 105.9 & 100.7 & 111.2 & 102.7 \\
\hline 28. Total consumption of Fixed Capital & LUNN & 101.3 & 100.0 & 96.3 & 92.1 & 91.6 & 91.1 & 91.7 & 88.6 & 87.3 & 92.7 & 88.7 \\
\hline Equipment & LUNI & 102.3 & 100.0 & 97.7 & 96.1 & 94.7 & 94.0 & 92.4 & 90.3 & 89.8 & 91.2 & 93.6 \\
\hline Buildings $s^{4,6}$ & LUNG & 104.2 & 100.0 & 101.3 & 103.3 & 100.6 & 98.3 & 94.9 & 96.7 & 93.5 & 102.3 & 101.3 \\
\hline Livestock & VQES & 94.3 & 100.0 & 88.1 & 74.6 & 77.0 & 78.2 & 85.0 & 77.2 & 76.2 & 83.8 & 72.5 \\
\hline Cattle & LUNJ & 88.1 & 100.0 & 86.4 & 76.0 & 81.0 & 81.5 & 92.2 & 79.1 & 75.1 & 84.0 & 66.6 \\
\hline Pigs & LUNL & 117.3 & 100.0 & 67.9 & 97.7 & 78.4 & 78.2 & 68.3 & 66.5 & 69.3 & 75.3 & 67.3 \\
\hline Sheep & LUNK & 95.1 & 100.0 & 120.7 & 78.7 & 78.0 & 78.7 & 86.2 & 86.7 & 85.1 & 88.6 & 83.3 \\
\hline Poultry & LUNM & 104.9 & 100.0 & 65.9 & 66.9 & 66.9 & 70.3 & 67.6 & 67.4 & 76.9 & 83.9 & 96.7 \\
\hline 29. Net value added at market prices & LUNO & 91.2 & 100.0 & 89.5 & 125.3 & 120.3 & 116.4 & 132.9 & 131.5 & 120.7 & 137.4 & 123.5 \\
\hline
\end{tabular}

1 See chapter text

5 Includes livestock and crop costs, water costs, insurance premiums, bank 2 Provisional. charges, professional fees, rates, and other farming costs.

3 Includes straw and minor crops

4 Landlords' expenses are included within 'Total maintenance', 'Other goods Source: Department for Environment, Food and Rural Affairs: 01904455080 and services' and 'Total consumption of Fixed Capital of buildings'. 


\begin{tabular}{|c|c|c|c|c|c|c|c|c|c|c|c|c|}
\hline & & 1999 & 2000 & 2001 & 2002 & 2003 & 2004 & 2005 & 2006 & 2007 & 2008 & 2009 \\
\hline Total agricultural area & $\mathrm{BFAH}$ & 18579 & 18311 & 18594 & 18537 & 18464 & 18432 & 18502 & 18788 & 18690 & 18702 & 18736 \\
\hline Total croppable land & JT3T & 6539.7 & 6494.7 & 6504.3 & 6460.5 & 6394.9 & 6423.0 & 6312.8 & 6197.3 & 6215.1 & 6070.5 & 6210.3 \\
\hline Crops & BFAA & 4709 & 4665 & 4493 & 4604 & 4475 & 4589 & 4437 & 4415 & 4439 & 4740 & 4694 \\
\hline Bare fallow ${ }^{2}$ & $\mathrm{BFAB}$ & 33 & 37 & 43 & 33 & 29 & 29 & 140 & 150 & 165 & & \\
\hline Uncropped arable land ${ }^{3}$ & J8U3 & 604.7 & 603.8 & 843.4 & 644.3 & 718.1 & 588.7 & 698.8 & 663.0 & 599.2 & 194.3 & 254.5 \\
\hline Total tillage & KIJR & 4742 & 4702 & 4536 & 4636 & 4504 & 4619 & 4600 & 4611 & 4603 & 4935 & \\
\hline All grass under 5 years old & KFEM & 1226.5 & 1225.6 & 1205.1 & 1242.6 & 1200.5 & 1245.8 & 1193.3 & 1137.1 & 1176.0 & 1141.0 & 1261.8 \\
\hline Total arable land & KFEN & 5968 & 5928 & 5741 & 5879 & 5705 & 5864 & 5794 & 5749 & 5779 & 6076 & \\
\hline All grasses 5 years old and over & KFEO & 5448.9 & 5363.1 & 5584.0 & 5518.6 & 5683.4 & 5620.2 & 5711.1 & 5967.3 & 5964.9 & 6035.6 & 6081.2 \\
\hline Total permanent grass & JT3V & 10023.6 & 9808.1 & 10018.6 & 10006.2 & 10012.7 & 9946.3 & 10065.0 & 10458.4 & 10283.8 & 10394.9 & 10244.7 \\
\hline Total tillage and grass & KFEP & 11417 & 11292 & 11325 & 11397 & 11388 & 11485 & 11505 & 11716 & 11744 & 12112 & \\
\hline Sole right rough grazing & BFAD & 4575 & 4445 & 4435 & 4488 & 4329 & 4326 & 4354 & 4491 & 4313 & 4359 & 4164 \\
\hline Set aside & DMNF & 572 & 567 & 800 & 612 & 689 & 559 & 535 & 513 & 440 & .. & \\
\hline All other land on agricultural holdings & & & & & & & & & & & & \\
\hline All other land & JT3X & .. & .. & 287.4 & 282.2 & 276.3 & 262.2 & 288.8 & 267.5 & 291.4 & 288.5 & 263.8 \\
\hline Total land on agricultural holdings & BFAF & 17352 & 17083 & 17361 & 17303 & 17227 & 17195 & 17266 & 17547 & 17452 & 17464 & 17498 \\
\hline Common rough grazing (estimated) & BFAG & 1227 & 1228 & 1232 & 1234 & 1236 & 1237 & 1236 & 1241 & 1238 & 1238 & 1238 \\
\hline Crops & BFAA & 4709 & 4665 & 4493 & 4604 & 4475 & 4589 & 4437 & 4415 & 4439 & 4740 & 4694 \\
\hline Arable crops & JT3U & 4529.7 & 4493.0 & 4283.2 & 4397.7 & 4300.6 & 4413.3 & 4251.0 & 4231.3 & 4271.3 & 4565.1 & 4521.6 \\
\hline Cereals & BFAJ & 3141 & 3348 & 3014 & 3245 & 3057 & 3130 & 2919 & 2864 & 2885 & 3274 & 3134 \\
\hline Wheat & BFAK & 1847 & 2086 & 1635 & 1996 & 1837 & 1990 & 1867 & 1836 & 1830 & 2080 & 1814 \\
\hline Barley & BFAL & 1179 & 1128 & 1245 & 1101 & 1076 & 1007 & 938 & 881 & 898 & 1032 & 1160 \\
\hline Oats & BFAM & 92 & 109 & 112 & 126 & 121 & 108 & 90 & 121 & 129 & 135 & 131 \\
\hline Mixed corn & BFAN & 2 & 2 & 3 & 4 & 4 & .. & .. & .. & .. & .. & .. \\
\hline Rye $^{4}$ & BFAO & 8 & 7 & 5 & 5 & 4 & 6 & .. & .. & .. & .. & .. \\
\hline Triticale & DMNH & 13 & 16 & 14 & 14 & 15 & 15 & 13 & 13 & 16 & - & .. \\
\hline Rye, mixed corn and triticale & J8U4 & 23 & 25 & 21 & 23 & 23 & 25 & 24 & 25 & 27 & 27 & 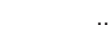 \\
\hline Other arable crops (excluding potatoes) & DMNI & 1211 & 979 & 1141 & 1024 & 1098 & 1136 & 1211 & 1245 & 1245 & 1152 & \\
\hline Oilseed rape & BFAP & 417 & 332 & 404 & 357 & 460 & 498 & 519 & 568 & 674 & 598 & 613 \\
\hline Sugar beet not for stock feeding ${ }^{4}$ & BFAQ & 183 & 173 & 177 & 169 & 162 & 154 & 148 & 130 & 125 & 120 & 116 \\
\hline Maize & JT3Y & 107.1 & 104.1 & 129.2 & 121.3 & 118.7 & 117.6 & 130.9 & 137.3 & 146.3 & 152.7 & 165.7 \\
\hline Other crops & DMNM & 197 & 192 & 214 & 204 & 201 & 203 & 252 & 278 & 272 & 269 & .. \\
\hline Potatoes & BFAR & 178 & 166 & 165 & 158 & 145 & 148 & 137 & 140 & 140 & 144 & 147 \\
\hline Horticultural & BFAV & 179 & 172 & 173 & 176 & 176 & 175 & 170 & 166 & 169 & 170 & 172 \\
\hline Vegetables grown in the open & DMNN & 126 & 119 & 120 & 124 & 125 & 125 & 121 & 119 & 121 & 122 & 124 \\
\hline Orchard fruit ${ }^{6}$ & BFBG & 28 & 28 & 28 & 26 & 25 & 24 & 23 & 23 & 23 & 24 & 24 \\
\hline Soft fruit & DMNO & 9 & 10 & 9 & 9 & 9 & 9 & 9 & 10 & 9 & 10 & 10 \\
\hline Ornamentals & DMNP & 13 & 14 & 14 & 15 & 14 & 15 & 14 & 12 & 13 & 13 & 12 \\
\hline Glasshouse crops & DMNQ & 2 & 2 & 2 & 2 & 2 & 2 & 2 & 2 & 2 & 2 & 2 \\
\hline
\end{tabular}

1 Includes estimates for minor holdings for all countries. See chapter text.

Source: Agricultural Departments: 01904455333

2 The area of bare fallow has shown an increase of 378\% in 2005. The rise in

the bare fallow area in England is believed to be due to the way the farmers have described their land following the introduction of the Single Payment Scheme.

3 Includes all uncropped arable land i.e. bare fallow and arable land not in production managed under GAEC12 conditions.

4 Figures are for England and Wales only.

5 Figures are for England only from 2005. From 2007 are included in Other

Crops.

6 Includes non-commercial orchards. 
Agriculture, fisheries and food

21.4 Estimated quantity of crops and grass harvested ${ }^{1}$

Thousand tonnes

\begin{tabular}{|c|c|c|c|c|c|c|c|c|c|c|c|c|}
\hline & & 1999 & 2000 & 2001 & 2002 & 2003 & 2004 & 2005 & 2006 & 2007 & 2008 & 2009 \\
\hline \multicolumn{13}{|l|}{ Agricultural crops } \\
\hline Wheat & BADO & 14867 & 16704 & 11580 & 15973 & 14282 & 15468 & 14863 & 14735 & 13221 & 17227 & 14379 \\
\hline Barley (Winter and Spring) & BADP & 6581 & 6492 & 6660 & 6128 & 6360 & 5799 & 5495 & 5239 & 5079 & 6144 & 6769 \\
\hline Oats & BADQ & 541 & 640 & 621 & 753 & 749 & 626 & 532 & 728 & 712 & 784 & 757 \\
\hline Sugar beet ${ }^{2}$ & BADR & 10584 & 9079 & 8335 & 9559 & 9168 & 8850 & 8687 & 7400 & 6733 & 7641 & 8330 \\
\hline \multirow[t]{2}{*}{ Potatoes } & BADS & 7131 & 6636 & 6649 & 6966 & 5918 & 6316 & 5979 & 5727 & 5564 & 6145 & 6423 \\
\hline & & 1999 & 2000 & 2001 & 2002 & 2003 & 2004 & 2005 & 2006 & 2007 & 2008 & 2009 \\
\hline \multirow{2}{*}{\multicolumn{13}{|c|}{$\begin{array}{l}\text { Horticultural crops } \\
\text { Field vegetables }\end{array}$}} \\
\hline & & & & & & & & & & & & \\
\hline Brussels sprouts & BADT & 72.5 & 78.5 & 67.3 & 54.8 & 42.7 & 55.8 & 45.1 & 46.1 & 44.8 & 43.3 & 43.6 \\
\hline \multicolumn{13}{|l|}{ Cabbage (including savoys } \\
\hline and spring greens) & BADU & 308.9 & 295.2 & 273.2 & 295.4 & 255.2 & 245.6 & 290.9 & 262.7 & 254.8 & 216.3 & 231.0 \\
\hline Cauliflowers & BADV & 191.7 & 172.4 & 156.1 & 107.4 & 116.5 & 126.3 & 168.3 & 133.2 & 123.7 & 122.1 & 108.5 \\
\hline Carrots & BADW & 617.6 & 673.2 & 725.8 & 760.0 & 718.4 & 602.4 & 671.1 & 710.1 & 701.3 & 735.4 & 671.0 \\
\hline Turnips and swedes & BADX & 117.5 & 123.3 & 132.1 & 141.8 & 103.9 & 96.5 & 97.0 & 103.1 & 105.7 & 106.2 & 116.5 \\
\hline Onions, dry bulb & BADZ & 342.0 & 391.4 & 392.7 & 374.9 & 283.4 & 373.6 & 340.9 & 413.6 & 358.8 & 303.8 & 354.9 \\
\hline \multicolumn{13}{|l|}{ Peas, green for market } \\
\hline (in pod weight) & BAEA & 7.0 & 7.0 & 6.7 & 6.2 & 7.2 & 5.9 & 5.9 & 5.9 & 5.9 & 5.9 & 5.9 \\
\hline \multicolumn{13}{|l|}{ Peas, green for processing } \\
\hline (shelled weight) & BAEB & 152.0 & 143.1 & 184.5 & 161.0 & 169.3 & 167.6 & 131.1 & 129.0 & 124.4 & 97.8 & 166.9 \\
\hline Lettuce & BAEC & 151.8 & 155.2 & 135.8 & 123.9 & 109.9 & 125.6 & 140.9 & 131.7 & 126.4 & 109.0 & 110.6 \\
\hline \multicolumn{13}{|l|}{ Protected crops } \\
\hline Tomatoes & BAED & 107.6 & 116.6 & 113.0 & 109.1 & 100.9 & 75.6 & 78.5 & 78.8 & 84.1 & 85.6 & 86.8 \\
\hline Cucumbers & BAEE & 83.8 & 83.8 & 79.8 & 71.5 & 73.6 & 77.0 & 61.4 & 59.9 & 56.5 & 49.4 & 57.7 \\
\hline Lettuce & BAEF & 20.6 & 19.9 & 18.7 & 20.9 & 16.0 & 16.6 & 10.4 & 8.1 & 8.2 & 7.8 & 6.8 \\
\hline \multicolumn{13}{|l|}{ Fruit } \\
\hline Dessert apples & BFCD & 97.8 & 133.9 & 101.3 & 104.4 & 84.0 & 69.0 & 92.2 & 118.0 & 129.3 & 106.2 & 121.1 \\
\hline Cooking apples & BFCE & 85.9 & 112.4 & 107.5 & 107.4 & 95.3 & 74.9 & 78.2 & 100.1 & 111.5 & 136.9 & 105.9 \\
\hline Soft fruit & BFCF & 60.1 & 65.9 & 65.6 & 64.6 & 67.1 & 79.9 & 86.0 & 105.4 & 107.7 & 124.1 & 150.6 \\
\hline Pears & BFBQ & 26.3 & 22.7 & 26.6 & 38.5 & 34.2 & 29.6 & 22.7 & 23.4 & 28.4 & 20.6 & 23.5 \\
\hline
\end{tabular}

1 See chapter text

Source: Agricultural Departments: 01904455332

2 Figures are adjusted to constant $16 \%$ sugar content. 


\section{Cattle, sheep, pigs and poultry on agricultural holdings ${ }^{1}$ \\ - United Kingdom}

At June each year

\begin{tabular}{|c|c|c|c|c|c|c|c|c|c|c|c|c|}
\hline & & 1999 & 2000 & 2001 & 2002 & 2003 & 2004 & 2005 & 2006 & 2007 & 2008 & 2009 \\
\hline Total cattle and calves ${ }^{2}$ & BFCG & 11423.4 & 11134.6 & 10602.1 & 10345.3 & 10508.2 & 10588.1 & 10770.2 & 10578.8 & 10303.9 & 10107.0 & 10025.5 \\
\hline \multicolumn{13}{|l|}{ of which: } \\
\hline dairy cows & $\mathrm{BFCH}$ & 2440.3 & 2335.8 & 2251.2 & 2227.2 & 2191.0 & 2128.8 & 1997.7 & 1978.8 & 1954.0 & 1908.9 & 1856.9 \\
\hline beef cows & BFCI & 1924.3 & 1842.2 & 1708.3 & 1657.0 & 1697.6 & 1736.0 & 1750.9 & 1737.1 & 1698.2 & 1670.2 & 1625.6 \\
\hline heifers in calf & BFCJ & 763 & 718 & 701 & 728 & 679 & 690 & 638 & 645 & .. & .. & \\
\hline \multicolumn{13}{|l|}{ of which: } \\
\hline ewes and shearlings & CKUQ & 21457.8 & 20448.6 & 17921.2 & 17630.1 & 17579.5 & 17630.4 & 16935.3 & 16636.9 & 16063.6 & 15616.2 & 14912.0 \\
\hline lambs under one year old & $\mathrm{BFCP}$ & 22092.1 & 20856.7 & 17768.9 & 17310.5 & 17322.0 & 17238.3 & 17487.6 & 17058.2 & 16855.4 & 16574.3 & 16177.4 \\
\hline Total pigs & $\mathrm{BFCQ}$ & 7283.9 & 6482.2 & 5845.4 & 5588.0 & 5045.8 & 5158.5 & 4861.9 & 4932.9 & 4834.4 & 4713.5 & 4724.3 \\
\hline \multicolumn{13}{|l|}{ of which: } \\
\hline gilts in pig & CKUR & 85.4 & 73.1 & 70.9 & 74.3 & 73.2 & 65.7 & 66.9 & 67.3 & 56.8 & 55.1 & 49.5 \\
\hline
\end{tabular}

Total fowls

KPSV 165156.9169772 .7179879 .7168996 .5178799 .5181759 .2173908 .7173080 .5167666 .9166199 .8159288 .1

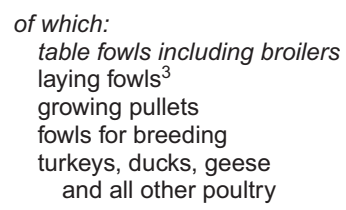

CKUT 101625.0105688 .8112530 .9105136 .6116737 .8119888 .3111474 .7110671 .91097942109858 .9102759 .1 CKUV 29257.928686 .929895 .228778 .029273 .929655 .229544 .128632 .527320 .825939 .826757 .4

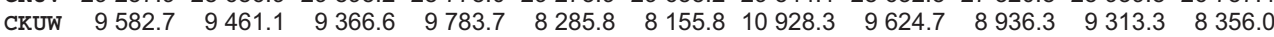

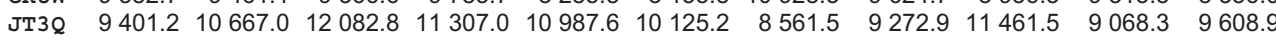

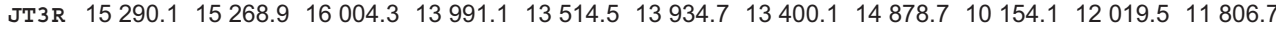

1 Includes estimates for minor holdings for all countries. See chapter text.

2 In 2007, cattle figures were sourced from the Cattle Tracing System (CTS)

in England and Wales, the equivalent APHIS system in Northern Ireland

Sources: Department for Environment, Food and Rural Affairs; and survey data in Scotland and are therefore not directly comparable with earlier years. To see comparable data for 2005-2007 please go to: http://statistics.defra.gov.uk/esg/statnot/june_uk.pdf

3 Excludes fowls laying eggs for hatching. 


\begin{tabular}{|c|c|c|c|c|c|c|c|c|c|c|c|c|}
\hline & & 198 & & 90 & 2000 & 2004 & 2005 & 2006 & & 07 & 2008 & 2009 \\
\hline Woodland area $^{2}$ - (Thousand hectares & & & & & & & & & & & & \\
\hline United Kingdom & $\mathrm{C} 5 \mathrm{OF}$ & 217 & & & 2793 & 2816 & 2825 & 2829 & & & 2841 & 2841 \\
\hline England $^{3}$ & C5OG & 94 & & 58 & 1103 & 1114 & 1119 & 1121 & & & 1127 & 1128 \\
\hline Wales ${ }^{3}$ & C50I & 24 & & 48 & 289 & 286 & 286 & 285 & & 85 & 285 & 284 \\
\hline Scotland ${ }^{3}$ & $\mathrm{C} 5 \mathrm{OH}$ & 92 & & & 1318 & 1330 & 1334 & 1337 & & & 1342 & 1341 \\
\hline Northern Ireland & C5OJ & 6 & & 74 & 83 & 86 & 85 & 86 & & 87 & 87 & \\
\hline Other ${ }^{5}$ & $\mathrm{C} 5 \mathrm{OL}$ & 123 & & & 1907 & 1974 & 1987 & 1997 & & & 2020 & 2027 \\
\hline Conifer & $\mathrm{C} 5 \mathrm{OM}$ & 137 & & 76 & 1663 & 1651 & 1647 & 1642 & & & 1635 & 1628 \\
\hline Broadleaved $^{6}$ & $\mathrm{C} 5 \mathrm{ON}$ & 80 & & 24 & 1131 & 1165 & 1178 & 1187 & & & 1207 & 1213 \\
\hline & & $\begin{array}{r}1998 \\
/ 99\end{array}$ & $\begin{array}{r}1999 \\
100\end{array}$ & $\begin{array}{r}2000 \\
/ 01\end{array}$ & $\begin{array}{r}2001 \\
/ 02\end{array}$ & $\begin{array}{r}2002 \\
103\end{array}$ & $\begin{array}{r}2003 \\
104\end{array}$ & $\begin{array}{r}2004 \\
105\end{array}$ & $\begin{array}{r}2005 \\
106\end{array}$ & $\begin{array}{r}2006 \\
107\end{array}$ & $\begin{array}{r}2007 \\
/ 08\end{array}$ & $\begin{array}{r}2008 \\
109\end{array}$ \\
\hline New Planting ${ }^{7}$ - (Thousand hectares) & & & & & & & & & & & & \\
\hline England & C5OP & 5.1 & 5.9 & 5.9 & 5.4 & 5.9 & 4.6 & 5.3 & 3.7 & 3.2 & 2.6 & \\
\hline Wales & C5OR & 0.6 & 0.7 & 0.4 & 0.3 & 0.3 & 0.5 & 0.5 & 0.5 & 0.4 & 0.2 & \\
\hline Scotland & C5OQ & 10.5 & 10.4 & 11.7 & 8.0 & 6.7 & 6.8 & 5.7 & 4.0 & 6.6 & 4.2 & \\
\hline Northern Ireland & C50s & 0.7 & 0.8 & 0.7 & 0.7 & 0.6 & 0.5 & 0.4 & 0.6 & 0.5 & 0.6 & \\
\hline Forestry Commission/Forest Service & С50T & 0.2 & 0.3 & 0.3 & 0.8 & 0.9 & 0.2 & 0.1 & 0.3 & 0.2 & 0.2 & \\
\hline Other $^{8}$ & C5OU & 16.8 & 17.6 & 18.4 & 13.6 & 12.6 & 12.1 & 11.8 & 8.4 & 10.4 & 7.4 & \\
\hline Conifer & $\mathrm{C} 5 \mathrm{OV}$ & 6.6 & 6.5 & 4.9 & 3.9 & 3.7 & 2.9 & 2.1 & 1.1 & 2.1 & 0.9 & \\
\hline Broadleaved & C50w & 10.4 & 11.4 & 13.8 & 10.5 & 9.8 & 9.5 & 9.8 & 7.6 & 8.5 & 6.7 & \\
\hline
\end{tabular}

Restocking ${ }^{7}$ - (Thousand hectares)

\begin{tabular}{|c|c|c|c|c|c|c|c|c|c|c|c|c|}
\hline United Kingdom & c50x & 14.1 & 15.2 & 15.3 & 13.9 & 14.5 & 14.9 & 16.1 & 15.9 & 19.0 & 18.9 & 15.9 \\
\hline England & C5OY & 4.1 & 3.9 & 4.0 & 3.4 & 3.4 & 3.2 & 2.8 & 3.2 & 2.8 & 3.5 & 3.2 \\
\hline Wales & C5P2 & 3.0 & 2.6 & 2.2 & 1.9 & 1.9 & 1.8 & 1.8 & 2.8 & 3.0 & 2.3 & 2.2 \\
\hline Scotland & $\mathrm{C} 50 \mathrm{z}$ & 6.3 & 8.0 & 8.0 & 7.8 & 8.5 & 8.9 & 10.4 & 9.0 & 12.4 & 12.6 & 9.6 \\
\hline Northern Ireland & C5P3 & 0.7 & 0.6 & 1.1 & 0.9 & 0.7 & 1.1 & 1.0 & 0.9 & 0.8 & 0.5 & 0.8 \\
\hline Forestry Commission/Forest Service & C5P4 & 8.5 & 8.8 & 8.9 & 9.2 & 9.1 & 9.9 & 10.6 & 10.4 & 11.0 & 10.4 & 9.2 \\
\hline Other $^{8}$ & C5P5 & 5.6 & 6.4 & 6.4 & 4.7 & 5.3 & 5.0 & 5.5 & 5.5 & 8.0 & 8.5 & 6.7 \\
\hline Conifer & C5P 6 & 11.3 & 11.9 & 12.3 & 11.5 & 12.0 & 12.1 & 13.0 & 12.5 & 15.3 & 14.8 & 12.1 \\
\hline Broadleaved & & & 2000 & 2001 & 2002 & 2003 & 2004 & & & 2006 & 2007 & 2008 \\
\hline
\end{tabular}

Wood Production (volume -

Thousand green tonnes ${ }^{9}$ )

\begin{tabular}{|c|c|c|c|c|c|c|c|c|c|c|}
\hline United Kingdom & C5P8 & 8080 & 8140 & 8250 & 8880 & 9040 & 9080 & 8950 & 9460 & 8850 \\
\hline Softwood total & C5PA & 7430 & 7500 & 7630 & 8320 & 8520 & 8490 & 8510 & 9020 & 42 \\
\hline Forestry Commission/Forest Service & C5 PB & 4850 & 4600 & 4650 & 4820 & 4890 & 4580 & 4580 & 4650 & 42 \\
\hline Non-Forestry Commission/Forest Service & C5PC & 2580 & 2900 & 2980 & 3500 & 3630 & 3910 & 3930 & 4360 & 00 \\
\hline
\end{tabular}

1 See chapter text.

6 Broadleaved includes coppice. For data based on 1979-82 Census, all scrub 2 Areas as at 31 March. and other non-plantation woodland have been assumed to be broadleaved.

3 For England, Wales and Scotland, 1980 woodland area figures are the pub- 7 Figures shown are for the areas of new planting and restocking in the year to lished results from the 1979-1982 Census of Woodlands and Trees and fig- 31 March.

ures for 1990 are adjusted to reflect subsequent changes. From 1998 on- 8 Includes grant aided planting on non-Forestry Commission/ Forest Service wards they are based on results from the 1995-1999 National Inventory of woodland and estimates for areas planted without the aid of grants.

Woodlands and Trees, adjusted to reflect subsequent changes. 9 Figures have been rounded to the nearest 10 thousand green tonnes.

4 The apparant fall in woodland cover in 2001 is due to the reclassification of 10 Hardwood is timber from broadleaved species. Most hardwood production in Forestry Commission open land within the forest. the UK comes from non-FC/FS woodland; the figures are estimates based on

5 Includes private woodland and non-Forestry Commission / Forest Service public woodland. 


\section{Sales for food of agricultural produce and livestock \\ 2. United Kingdom}

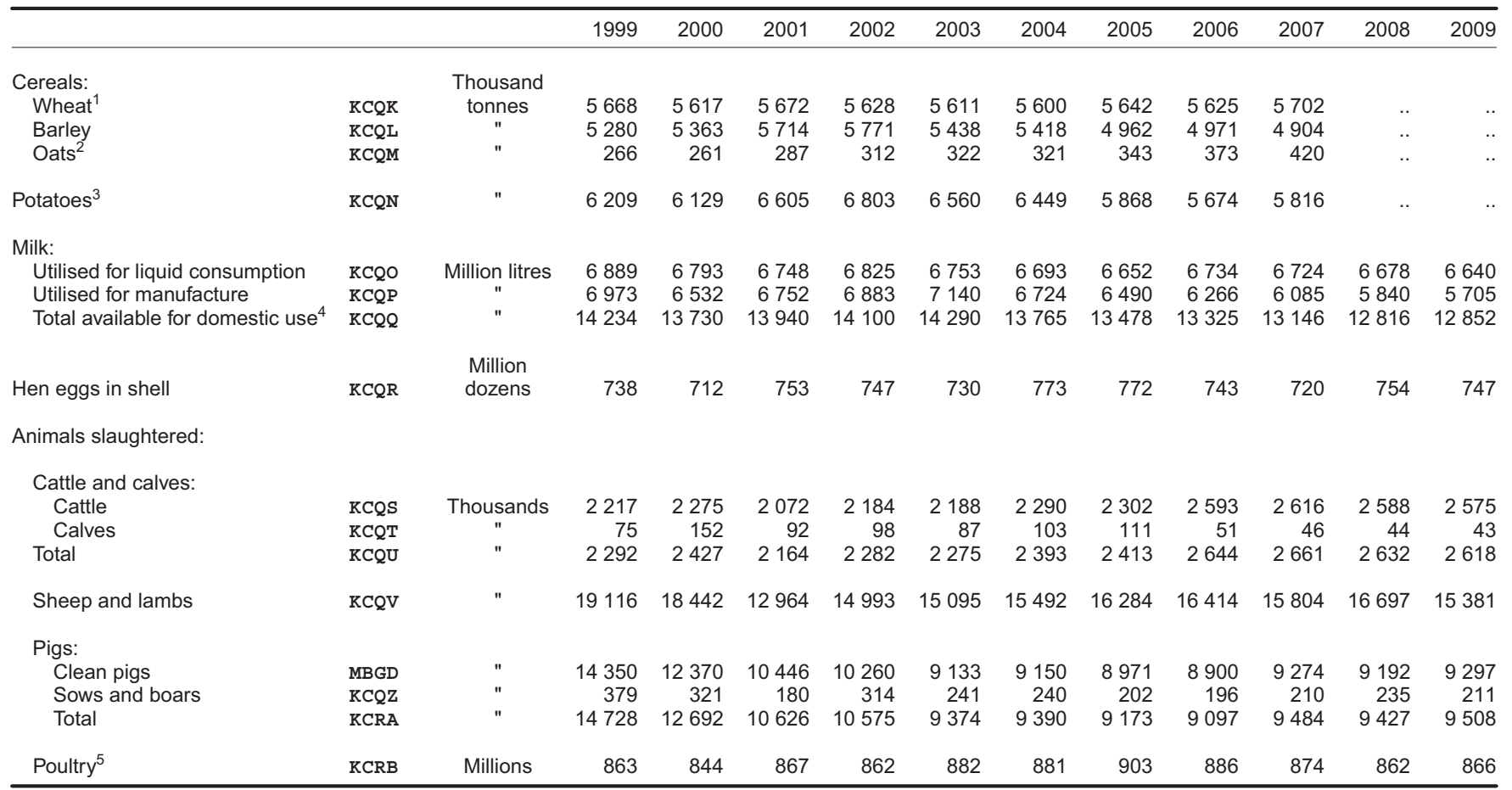

Note: The figures for cereals and for animals slaughtered relate to periods 4 The totals of liquid consumption and milk used for manufacture may not add up of 52 weeks

to the total available for domestic use because of adjustments for dairy wastage, stock changes and other uses, such as farmhouse consumption, milk

1 Flour millers' receipts of home-grown wheat.

2 Oatmeal millers' receipts of home-grown oats.

3 Total sales for human consumption in the UK. Data for 2007 are provisional. fed to stock and on farm waste.

5 Total fowls, ducks, geese and turkeys.

Source: Department for Environment, Food and Rural Affairs: 01904455333

\section{Estimates of producers of organic and in-conversion livestock ${ }^{1}$ 21.0 United Kingdom}

Thousand head

\begin{tabular}{|c|c|c|c|c|c|c|}
\hline & & 2004 & 2005 & 2006 & 2007 & 2008 \\
\hline Cattle & IDR 8 & 174.8 & 214.3 & 244.8 & 250.4 & 319.6 \\
\hline Sheep & IDR 9 & 571.6 & 691.0 & 747.3 & 863.1 & 1178.3 \\
\hline Pigs & IDS2 & 43.7 & 30.0 & 32.9 & 50.4 & 71.2 \\
\hline Poultry & IDS3 & 2431.6 & 3439.5 & 4421.3 & 4440.7 & 4362.9 \\
\hline Goats & IDS4 & 0.5 & 0.5 & 0.6 & 0.5 & 0.4 \\
\hline Other Livestock & IDS5 & 1.2 & 1.5 & 4.3 & 3.4 & 4.4 \\
\hline
\end{tabular}

Note: DEFRA have recalculated the basis on which these data are collected to make it clearer that they are an average for the year(see footnote 1).

1 Certification bodies record production data at various times of the year so

figures should be treated with care as they will not represent an exact snap-

shot of organic livestock farming. 
Agriculture, fisheries and food

Producers of organic and in-conversion livestock, Organic producers,

growers, processors and importers

United Kingdom

Number of producers or businesses

\begin{tabular}{|c|c|c|c|c|c|c|}
\hline & & 2004 & 2005 & 2006 & 2007 & 2008 \\
\hline \multicolumn{7}{|c|}{ Producers of organic and in-conversion livestock } \\
\hline North East & IDZ2 & 49 & 44 & 54 & 46 & 58 \\
\hline North West & IDZ3 & 122 & 87 & 102 & 104 & 95 \\
\hline Yorkshire and Humberside & IDZ4 & 82 & 54 & 82 & 82 & 85 \\
\hline East Midlands & IDZ5 & 135 & 110 & 125 & 121 & 127 \\
\hline West Midlands & IDZ6 & 196 & 162 & 196 & 174 & 190 \\
\hline Eastern & IDZ7 & 99 & 69 & 86 & 91 & 76 \\
\hline South West & IDZ8 & 761 & 553 & 724 & 706 & 705 \\
\hline England & IE22 & 1664 & 1241 & 570 & 1503 & 1524 \\
\hline Wales & IE23 & 469 & 402 & 502 & 493 & 580 \\
\hline Scotland & IE24 & 385 & 293 & 296 & 285 & 282 \\
\hline Northern Ireland & IE25 & 119 & 110 & 140 & 167 & 176 \\
\hline \multirow[t]{2}{*}{ United Kingdom } & IE26 & 2637 & 2046 & 2508 & 2448 & 2562 \\
\hline & & 2004 & 2005 & 2006 & 2007 & 2008 \\
\hline North West & IE2 8 & 169 & 176 & 168 & 173 & 211 \\
\hline Yorkshire and Humberside & IE29 & 134 & 149 & 138 & 155 & 190 \\
\hline East Midlands & $I E 2 \mathrm{~A}$ & 218 & 237 & 221 & 236 & 276 \\
\hline West Midlands & $I E 2 B$ & 325 & 337 & 335 & 351 & 408 \\
\hline Eastern & IE $2 \mathrm{C}$ & 258 & 259 & 253 & 267 & 315 \\
\hline South West & IE2D & 1020 & 1123 & 1152 & 1282 & 1631 \\
\hline South East (including London) & IE2 $\mathrm{E}$ & 409 & 463 & 417 & 423 & 556 \\
\hline England & IE2 F & 2607 & 2827 & 2785 & 3003 & 3724 \\
\hline Wales & IE $2 \mathrm{G}$ & 623 & 667 & 688 & 710 & 857 \\
\hline Scotland & $\mathrm{IE} 2 \mathrm{H}$ & 689 & 653 & 595 & 686 & 671 \\
\hline Northern Ireland & IE2I & 153 & 174 & 217 & 240 & 254 \\
\hline United Kingdom & IE2 J & 4072 & 4321 & 4285 & 4639 & 5506 \\
\hline \multicolumn{7}{|c|}{ Processors and/or importers businesses ${ }^{1}$} \\
\hline North East & $\mathrm{IE} 2 \mathrm{~K}$ & 31 & 19 & 28 & 45 & 53 \\
\hline North West & IE2L & 130 & 107 & 143 & 159 & 180 \\
\hline Yorkshire and Humberside & IE $2 \mathrm{M}$ & 126 & 121 & 141 & 164 & 191 \\
\hline South West & IE $2 Q$ & 353 & 242 & 380 & 450 & 509 \\
\hline South East (including London) & IE2 R & 450 & 387 & 484 & 516 & 579 \\
\hline England & $\mathrm{IE} 2 \mathrm{~S}$ & 1669 & 1353 & 1769 & 2002 & 2239 \\
\hline Wales & IE2T & 112 & 85 & 112 & 125 & 149 \\
\hline Scotland & IE $2 U$ & 174 & 152 & 197 & 225 & 231 \\
\hline Northern Ireland & $I E 2 \mathrm{~V}$ & 35 & 36 & 50 & 52 & 56 \\
\hline United Kingdom & IE2W & 1990 & 1626 & 2128 & 2404 & 2675 \\
\hline
\end{tabular}

Note: DEFRA have recalculated the basis on which these data are collected to make it clearer that they are an average for the year(see footnote 1).

1 Processors and importers include abattoirs, bakers, stores and whole-

Sources: Department for Environment, Food and Rural Affairs Organic Statistics Team:01904 455558

salers. The recorded location depends on the address registered with the

Sector Bodies and so larger businesses may be recorded at their head-

quarters. 


\section{Organic and in-conversion land and land use 21.10 Unitod Kingsom}

Thousand hectare

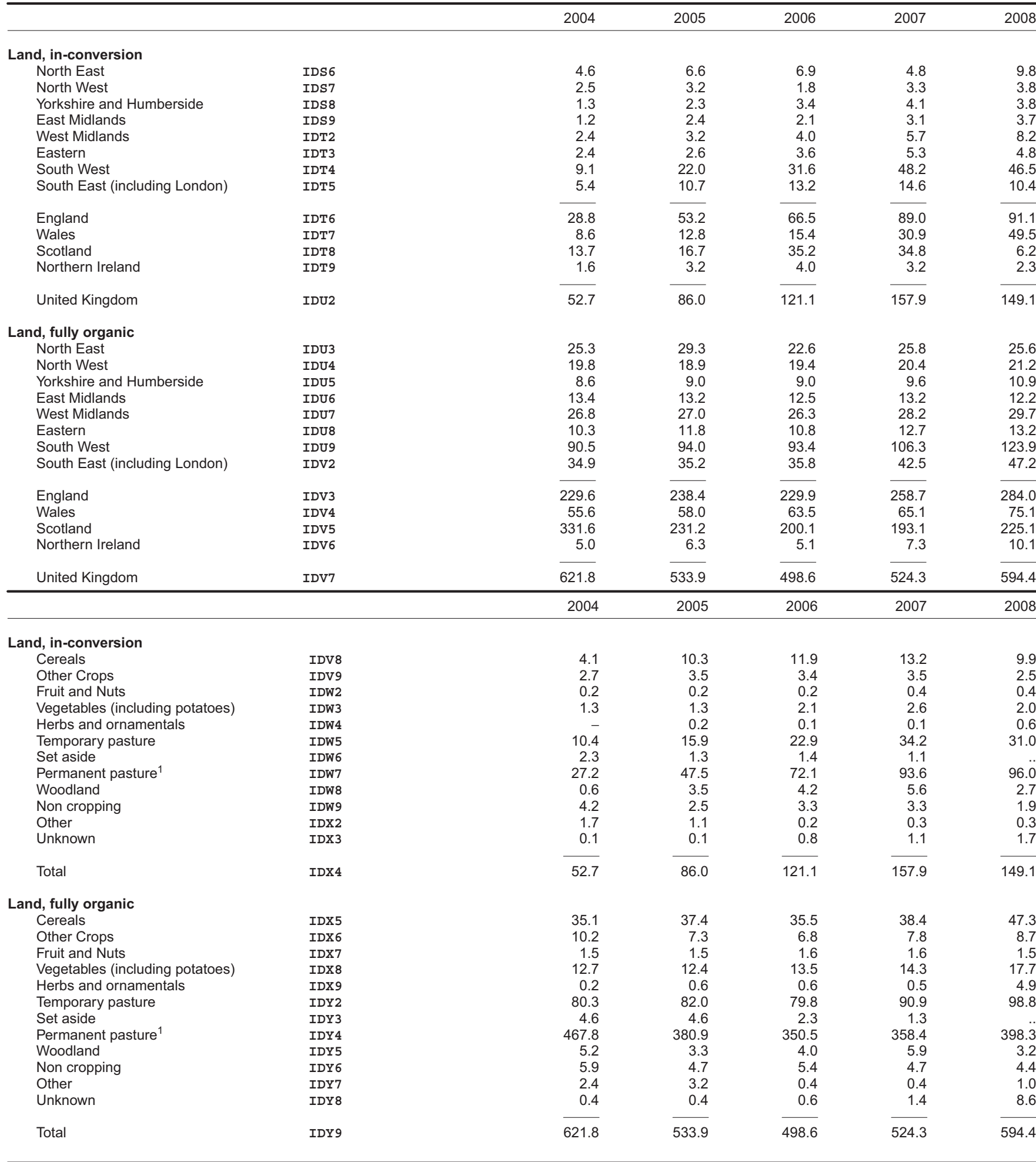

Note: DEFRA have recalculated the basis on which these data are collected to make it clearer that they are a yearly average, not a snapshot. 
England and Wales: At September each year

\begin{tabular}{|c|c|c|c|c|c|c|c|}
\hline & & 2004 & 2005 & 2006 & 2007 & 2008 & 2009 \\
\hline $\begin{array}{l}\text { Average weekly earnings }(£) \\
95 \% \text { confidence interval }\end{array}$ & LQML & $\begin{array}{r}331.26 \\
(+/-£ 12.46)\end{array}$ & $\begin{array}{r}357.64 \\
(+/-£ 13.62)\end{array}$ & $\begin{array}{r}340.60 \\
(+/-£ 22.05)\end{array}$ & $\begin{array}{r}352.33 \\
(+/-£ 13.62)\end{array}$ & $\begin{array}{r}356.13 \\
(+/-£ 18.97)\end{array}$ & $\begin{array}{r}404.05 \\
(+/-£ 19.93)\end{array}$ \\
\hline $\begin{array}{l}\text { Average weekly hours worked } \\
95 \% \text { confidence interval }\end{array}$ & LQMM & $\begin{array}{r}46.2 \\
(+/-1.2)\end{array}$ & $\begin{array}{r}48.4 \\
(+/-1.4)\end{array}$ & $\begin{array}{r}46.1 \\
(+/-2.4)\end{array}$ & $\begin{array}{r}47.0 \\
(+/-1.4)\end{array}$ & $\begin{array}{r}46.6 \\
(+/-2.3)\end{array}$ & $\begin{array}{r}49.4 \\
(+/-2.1)\end{array}$ \\
\hline $\begin{array}{l}\text { Average earnings/hours }(£) \\
95 \% \text { confidence interval }\end{array}$ & LQMN & $\begin{array}{r}7.16 \\
(+/-£ 0.17)\end{array}$ & $\begin{array}{r}7.40 \\
(+/-£ 0.17)\end{array}$ & $\begin{array}{r}7.39 \\
(+/-£ 0.21)\end{array}$ & $\begin{array}{r}7.50 \\
(+/-£ 0.16)\end{array}$ & $\begin{array}{r}7.64 \\
(+/-£ 0.16)\end{array}$ & $\begin{array}{r}8.19 \\
(+/-£ 0.17)\end{array}$ \\
\hline Number of workers in the sample & & 311 & 299 & 248 & 279 & 283 & 231 \\
\hline
\end{tabular}

\section{Average weekly and hourly earnings and hours of agricultural workers ${ }^{1}$ : by type, aged 20 and over}

England and Wales: At September 2009

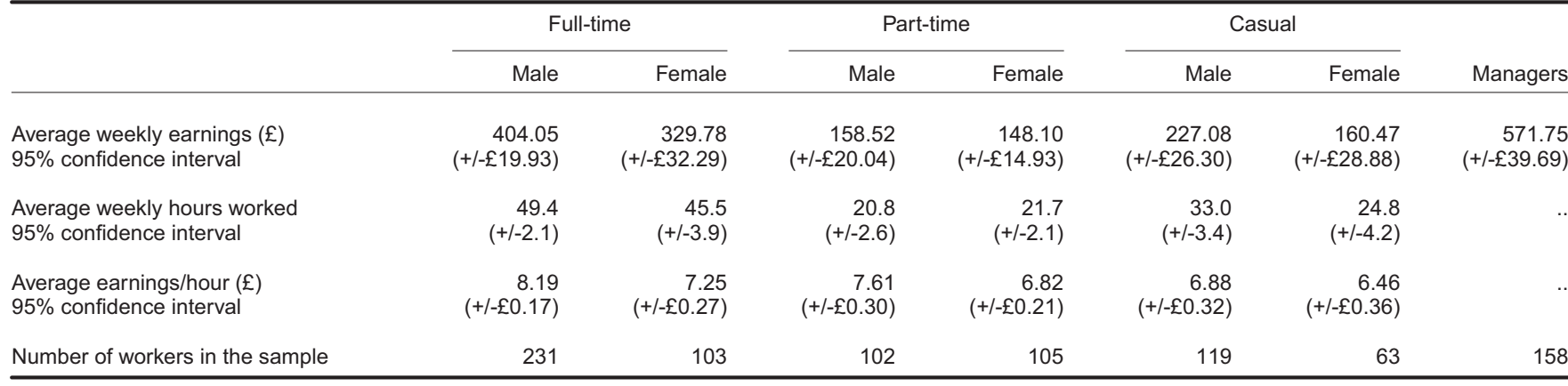

1 See chapter text.

\section{Workers employed in agriculture ${ }^{1,2}$ : by type \\ 21.13 Unites Kingsom \\ At June each year}

Thousands

\begin{tabular}{|c|c|c|c|c|c|c|c|c|c|c|c|c|}
\hline & \multicolumn{5}{|c|}{ Regular } & \multicolumn{3}{|c|}{ Seasonal or casual } & \multicolumn{3}{|c|}{ All } & \multirow{3}{*}{$\begin{array}{r}\text { Salaried } \\
\text { managers }\end{array}$} \\
\hline & \multirow[b]{2}{*}{ Total } & \multicolumn{2}{|c|}{ Full - time } & \multicolumn{2}{|c|}{ Part - time ${ }^{6}$} & \multirow[b]{2}{*}{ Total } & \multirow[b]{2}{*}{ Male } & \multirow[b]{2}{*}{ Female } & \multirow[b]{2}{*}{ Total } & \multirow[b]{2}{*}{ Male } & \multirow[b]{2}{*}{ Female } & \\
\hline & & Male & Female & Male & Female & & & & & & & \\
\hline & BANC & BAMY & BAMZ & BANA & BANB & BANF & BAND & BANE & BANI & BANG & BANH & KAYG \\
\hline 1995 & 157.4 & 90.4 & 13.0 & 30.0 & 24.1 & 83.7 & 56.5 & 27.2 & 241.2 & 176.8 & 64.3 & 7.7 \\
\hline 1996 & 156.4 & 89.2 & 12.6 & 31.2 & 23.4 & 81.5 & 55.6 & 25.8 & 237.9 & 176.0 & 61.9 & 7.8 \\
\hline 1997 & 154.4 & 87.5 & 12.6 & 31.2 & 23.1 & 80.9 & 55.3 & 25.5 & 235.2 & 174.0 & 61.2 & 7.8 \\
\hline $1998^{3,4}$ & 155.6 & 88.0 & 13.1 & 29.7 & 24.7 & 79.5 & 55.6 & 23.8 & 235.0 & 172.8 & 62.2 & 12.1 \\
\hline 1999 & 144.7 & 82.7 & 11.9 & 27.5 & 22.6 & 73.0 & 51.8 & 21.2 & 217.7 & 162.0 & 55.6 & 13.8 \\
\hline 2000 & 128.9 & 73.4 & 10.3 & 24.6 & 20.6 & 64.4 & 45.9 & 18.5 & 193.3 & 143.9 & 49.4 & 11.1 \\
\hline $2001^{5}$ & 120.8 & $\underline{69.0}$ & 10.9 & $\underline{22.0}$ & 18.9 & 63.2 & 44.6 & $\underline{18.6}$ & 184.0 & 135.6 & $\underline{48.5}$ & 13.4 \\
\hline$\angle 001$ & 123.5 & 70.3 & 11.2 & 22.5 & 19.4 & 64.1 & 45.4 & 18.8 & 187.6 & 138.2 & 49.4 & 14.1 \\
\hline 2002 & 116.3 & 64.7 & 11.5 & 21.7 & 18.4 & 64.2 & 46.2 & 18.0 & 180.6 & 132.6 & 47.9 & 13.4 \\
\hline 2003 & 108.4 & 60.4 & 10.0 & 21.0 & 17.0 & 62.6 & 44.8 & 17.8 & 170.9 & 126.2 & 44.8 & 12.7 \\
\hline 2005 Jun & 109.2 & 57.2 & 10.3 & 24.5 & 17.2 & 65.1 & 46.4 & 18.7 & 174.3 & 128.1 & 46.2 & 15.7 \\
\hline 2006 Jun & 105.4 & 53.6 & 10.4 & 24.3 & 17.1 & 64.0 & 44.4 & 19.6 & 169.4 & 122.3 & 47.1 & 14.6 \\
\hline 2007 Jun & 107.8 & 52.2 & 10.3 & 28.0 & 17.3 & 58.9 & 41.0 & 17.9 & 166.6 & 121.2 & 45.5 & 15.4 \\
\hline 2008 Jun & 111.1 & 54.7 & 11.3 & 27.9 & 17.2 & 61.7 & 43.2 & 18.6 & 172.8 & 125.8 & 47.1 & 15.1 \\
\hline 2009 Jun & 113.1 & 54.5 & 11.7 & 29.0 & 17.9 & 61.5 & 43.5 & 18.1 & 174.7 & 127.0 & 47.7 & 12.2 \\
\hline
\end{tabular}

1 See chapter text. Includes estimates for minor holdings for all countries. 4 From 1998, all farmers managing holdings for limited companies or other insti-

2 Figures exclude schoolchildren but include trainees employed under an offi-

tutions in England and Wales were asked to classify themselves as salaried

cial youth training scheme and paid at Agricultural Wages Board rates or

above.

managers.

5 Due to an English register improvement only the top figure for 2001 is directly comparable with 2000 , while the bottom figure for 2001 is only comparable with data from 2002

(n) 1998 onwards are not comparable with previous years, due to

Census in England, Wales and Scotland.

6 Part time is defined as less than 39 hours per week in England and Wales, less than 38 hours per week in Scotland and less than 30 hours per week in Northern Ireland. 
$£$ million (unless otherwise stated)

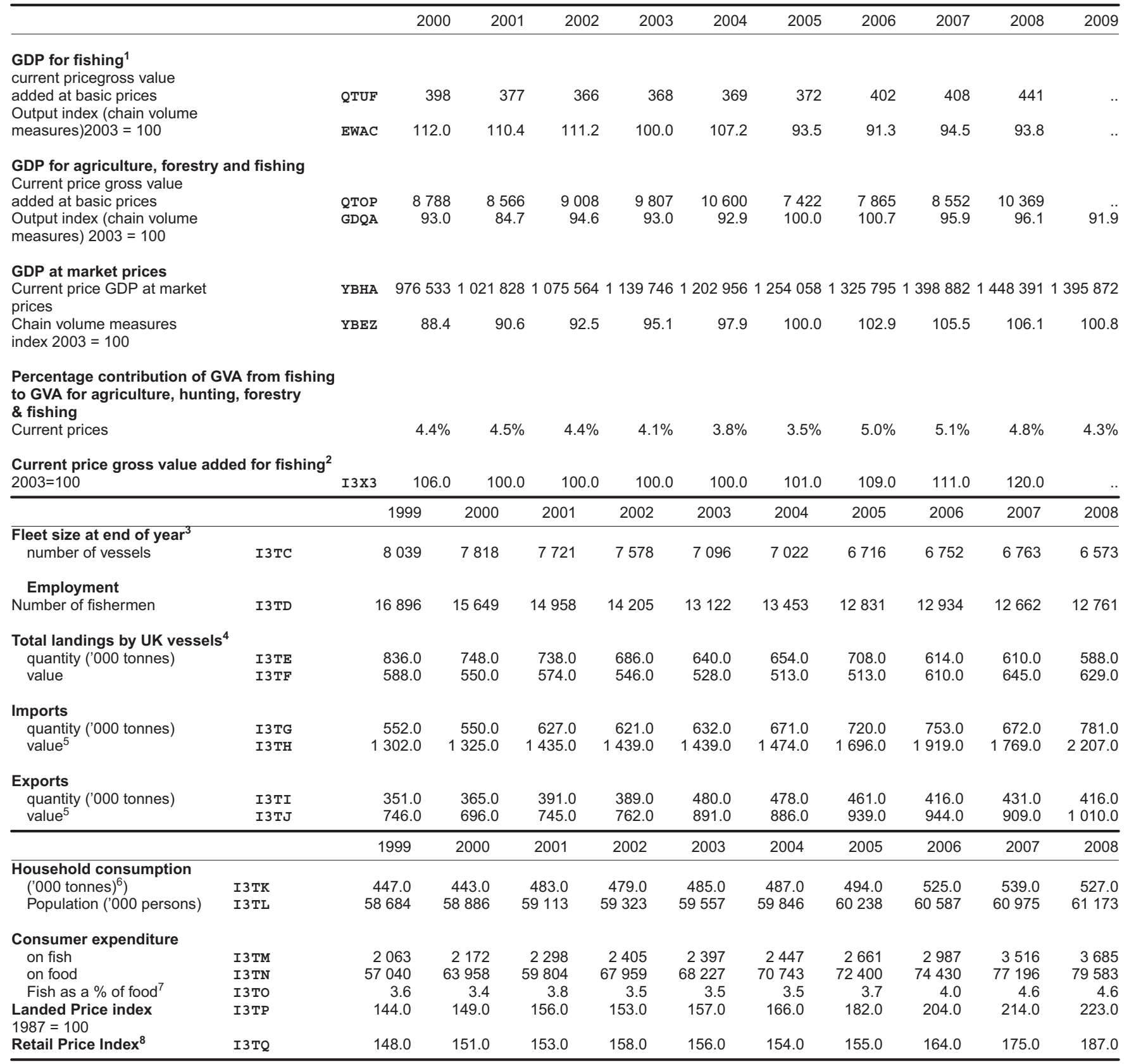

1 GDP for fish includes landings abroad.

5 Imports are valued at cost, including insurance and freight terms whereas ex-

2 Year on year comparisons may be affected by changes in the industrial ports are valued at free on board terms.

classification of some contributors. For most businesses data are appropri- 6 Data are derived from the National Food Survey prior to 2001, and from the ate to a single activity heading; where information covers a mixture of activi- $\quad$ Expenditure and Food Survey from 2001 onwards. Figures for 2001 onwards ties, the business is classified according to the main activity. are based on financial year data.

3 The number of vessels includes those registered in the Channel Islands 7 Including non-alcoholic beverages

and the Isle of Man.

4 The quantity of landed fish is expressed in terms of liveweight. The figures

8 The fish component of the RPI which includes canned and processed fish. The relate to landings both into the UK and abroad.

index is calculated on a monthly basis with January $1987=100$. 
Agriculture, fisheries and food

$245 \begin{aligned} & \text { Fishing fleet }{ }^{1} \\ & \text { United Kingdom }\end{aligned}$

At 31 December each year

Numbers

\begin{tabular}{|c|c|c|c|c|c|c|c|c|c|c|c|c|}
\hline & & 1999 & 2000 & 2001 & 2002 & 2003 & 2004 & 2005 & 2006 & 2007 & 2008 & 2009 \\
\hline \multicolumn{13}{|l|}{ By size } \\
\hline $10 \mathrm{~m}$ and under & KSNF & 5409 & 5273 & 5227 & 5287 & 5113 & 5092 & 4833 & 4896 & 4521 & 4520 & 4435 \\
\hline $10.01-12.19 m$ & KSNG & 577 & 547 & 536 & 514 & 486 & 465 & 449 & 445 & 446 & 439 & 430 \\
\hline $12.20-17.00 m$ & $\mathrm{KSNH}$ & 468 & 467 & 442 & 409 & 405 & 393 & 387 & 384 & 378 & 379 & 372 \\
\hline $17.01-18.29 m$ & KSNI & 154 & 131 & 143 & 129 & 121 & 115 & 112 & 111 & 110 & 106 & 102 \\
\hline $18.30-24.38 m$ & KSNJ & 414 & 406 & 405 & 322 & 271 & 257 & 253 & 244 & 245 & 247 & 248 \\
\hline $24.39-30.48 m$ & KSNK & 224 & 219 & 218 & 185 & 156 & 147 & 143 & 139 & 121 & 125 & 126 \\
\hline $30.49-36.58 m$ & KSNL & 80 & 77 & 75 & 65 & 63 & 60 & 55 & 56 & 44 & 41 & 43 \\
\hline over $36.58 \mathrm{~m}$ & KSNM & 122 & 122 & 123 & 122 & 120 & 112 & 109 & 97 & 88 & 79 & 73 \\
\hline Total over $10 \mathrm{~m}$ & KSNN & 2039 & 1969 & 1942 & 1746 & 1622 & 1549 & 1508 & 1476 & 1432 & 1416 & 1394 \\
\hline Total UK fleet ${ }^{2}$ & KSNO & 7448 & 7242 & 7169 & 7033 & 6735 & 6641 & 6341 & 6372 & 5953 & 5936 & 5829 \\
\hline \multicolumn{13}{|l|}{ By segment } \\
\hline Pelagic gears & KSNP & 46 & 44 & 47 & 45 & 42 & 31 & 23 & 16 & .. & .. & .. \\
\hline Beam trawl & KSNQ & 114 & 111 & 116 & 113 & 162 & 102 & 96 & 93 & .. & .. & .. \\
\hline Demersal, Seines and Nephrops & JZCI & 1235 & 1208 & 1158 & 969 & 853 & 852 & 812 & 785 & .. & .. & .. \\
\hline Lines and Nets & KSNR & 172 & 165 & 146 & 136 & 118 & 123 & 114 & 111 & .. & .. & .. \\
\hline Shellfish: mobile & KSNS & 243 & 211 & 229 & 228 & 191 & 166 & 155 & 151 & .. & .. & .. \\
\hline Shellfish: fixed & KSNT & 301 & 297 & 301 & 304 & 307 & 253 & 236 & 230 & .. & .. & .. \\
\hline Distant water & KSNU & 12 & 13 & 11 & 10 & 8 & 10 & 10 & 8 & .. & .. & .. \\
\hline Under 10m & KSNV & 5916 & 5769 & 5713 & 5773 & 5587 & 5395 & 4276 & 4131 & .. & .. & .. \\
\hline Other: Mussel Dredgers & JZCJ & 2 & 2 & 7 & 15 & 15 & 13 & 7 & 6 & .. & .. & .. \\
\hline Total UK fleet ${ }^{3}$ & I3TC & 8039 & 7818 & 7721 & 7578 & 7096 & 7022 & 6716 & 6752 & 6763 & 6573 & \\
\hline
\end{tabular}

1 See chapter text.

Source: Fisheries Statistics Unit:020 72728096

2 Excluding Channel Islands and Isle of Man.

3 Including Channel Islands and Isle of Man. 
Grammes per person per week

\begin{tabular}{|c|c|c|c|c|c|c|c|c|c|c|c|c|c|c|}
\hline & & \multicolumn{4}{|c|}{ Great Britain } & & \multicolumn{8}{|c|}{ United Kingdom } \\
\hline & & 1997 & 1998 & 1999 & 2000 & & $\begin{array}{r}2001 \\
102\end{array}$ & $\begin{array}{r}2002 \\
103\end{array}$ & $\begin{array}{r}2003 \\
104\end{array}$ & $\begin{array}{r}2004 \\
105\end{array}$ & $\begin{array}{r}2005 \\
106\end{array}$ & 2006 & 2007 & 2008 \\
\hline Liquid wholemilk² (ml) & KPQM & 712 & 693 & 634 & 664 & VQEW & 599 & 555 & 585 & 484 & 460 & 477 & 420 & 410 \\
\hline Fully skimmed (ml) & KZBH & 158 & 164 & 167 & 164 & VQEX & 160 & 166 & 154 & 158 & 159 & 163 & 173 & 158 \\
\hline Semi skimmed (ml) & KZBI & 978 & 945 & 958 & 975 & VQEZ & 931 & 919 & 926 & 975 & 1008 & 974 & 982 & 987 \\
\hline Other milk and cream (ml) & KZBJ & 248 & 243 & 248 & 278 & VQFA & 333 & 350 & 358 & 366 & 385 & 395 & 397 & 392 \\
\hline Cheese & $\mathrm{KPQO}$ & 109 & 104 & 104 & 110 & VQFB & 112 & 112 & 113 & 110 & 116 & 116 & 119 & 111 \\
\hline Butter & KPQP & 38 & 39 & 37 & 39 & VQFC & 42 & 37 & 35 & 35 & 38 & 40 & 41 & 40 \\
\hline Margarine & KPQQ & 26 & 26 & 20 & 21 & VQFD & 13 & 13 & 12 & 11 & 20 & 18 & 19 & 22 \\
\hline Low and reduced fat spreads & KZBK & 77 & 69 & 71 & 68 & VQFE & 72 & 70 & 71 & 68 & 55 & 57 & 53 & 51 \\
\hline All other oils and fats ( $\mathrm{ml}$ for oils) & $\mathrm{KPQR}$ & 62 & 62 & 58 & 58 & VQFF & 70 & 70 & 68 & 68 & 70 & 69 & 68 & 72 \\
\hline Eggs (number) & KPQS & 2 & 2 & 2 & 2 & VQFG & 2 & 2 & 2 & 2 & 2 & 2 & 2 & 2 \\
\hline Preserves and honey & KPQT & 41 & 38 & 33 & 33 & VQFH & 35 & 34 & 33 & 34 & 35 & 34 & 33 & 34 \\
\hline Sugar & KPQU & 128 & 119 & 107 & 105 & VQFI & 112 & 111 & 102 & 99 & 94 & 92 & 92 & 93 \\
\hline Beef and veal & $\mathrm{KPQV}$ & 110 & 109 & 110 & 124 & VQFJ & 118 & 118 & 119 & 123 & 120 & 128 & 126 & 111 \\
\hline Mutton and lamb & KPQW & 56 & 59 & 57 & 55 & VQFK & 51 & 51 & 49 & 50 & 53 & 54 & 55 & 45 \\
\hline Pork & KPQXX & 75 & 76 & 69 & 68 & VQFL & 61 & 61 & 56 & 56 & 52 & 55 & 54 & 55 \\
\hline $\begin{array}{l}\text { Bacon and ham, uncooked } \\
\text { Bacon and ham, cooked (includ }\end{array}$ & KPQY & 72 & 76 & 68 & 71 & VQFM & 68 & 69 & 70 & 70 & 68 & 66 & 64 & 63 \\
\hline canned) & KPQZ & 41 & 40 & 39 & 41 & VQFN & 45 & 45 & 47 & 43 & 44 & 45 & 45 & 45 \\
\hline Poultry uncooked & $\mathrm{JZCH}$ & 221 & 218 & 201 & 214 & VQFO & 206 & 199 & 200 & 197 & 212 & 207 & 208 & 207 \\
\hline Cooked poultry (not purchased in cans) & KYBP & 33 & 33 & 35 & 39 & $\mathrm{VQFQ}$ & 44 & 45 & 48 & 49 & 48 & 48 & 43 & 44 \\
\hline Other cooked and canned meats & KPRB & 52 & 49 & 48 & 51 & VQFR & 54 & 59 & 60 & 58 & 56 & 53 & 50 & 51 \\
\hline Offals & KPRC & 7 & 5 & 5 & 5 & VQFS & 6 & 6 & 7 & 5 & 5 & 5 & 5 & 5 \\
\hline Sausages, uncooked & KPRD & 63 & 60 & 58 & 60 & VQFT & 66 & 66 & 70 & 67 & 64 & 65 & 65 & 62 \\
\hline Other meat products & KPRE & 209 & 216 & 221 & 239 & VQFU & 313 & 319 & 335 & 330 & 323 & 315 & 316 & 311 \\
\hline Fish, fresh and processed & & & & & & & & & & & & & & \\
\hline (including shellfish) & KPRF & 70 & 70 & 70 & 67 & & & & & & & & & \\
\hline Canned fish & KPRG & 31 & 29 & 31 & 32 & & & & & & & & & \\
\hline Fish and fish products, frozen & KPRH & 46 & 46 & 42 & 44 & & & & & & & & & \\
\hline Fish, fresh chilled or frozen & & & & & & VQAI & 51 & 48 & 45 & 42 & 45 & 47 & 43 & 43 \\
\hline Other fish and fish products & & & & & & VQAJ & 105 & 106 & 111 & 115 & 122 & 123 & 122 & 118 \\
\hline Fresh green vegetables & KPRJ & 251 & 246 & 245 & 240 & VQAK & 229 & 231 & 228 & 225 & 235 & 221 & 224 & 203 \\
\hline Other fresh vegetables & KPRK & 497 & 486 & 500 & 492 & VQAL & 502 & 505 & 505 & 536 & 567 & 566 & 566 & 557 \\
\hline Frozen potato products & KYBQ & 106 & 111 & 113 & 120 & & & & & & & & & \\
\hline Other frozen vegetables & KPRL & 94 & 88 & 87 & 80 & & & & & & & & & \\
\hline Potato products not frozen & JZCF & 90 & 89 & 86 & 82 & & & & & & & & & \\
\hline Canned beans & KPRM & 122 & 118 & 112 & 114 & & & & & & & & & \\
\hline Other canned vegetables (excl. potatoes) & KPRN & 104 & 99 & 92 & 97 & & & & & & & & & \\
\hline Other processed vegetables (excl. potatoes) & $\mathrm{LQZH}$ & 52 & 54 & 59 & 54 & & & & & & & & & \\
\hline All processed vegetables & & & & & & VQAM & 620 & 613 & 611 & 597 & 608 & 601 & 594 & 599 \\
\hline Apples & KPRO & 179 & 181 & 169 & 180 & VQGN & 175 & 172 & 171 & 173 & 179 & 180 & 178 & 162 \\
\hline Bananas & KPRP & 195 & 198 & 202 & 206 & VQिGO & 203 & 208 & 211 & 217 & 225 & 226 & 230 & 219 \\
\hline Oranges & KPRQ & 62 & 63 & 50 & 54 & VQGP & 55 & 62 & 64 & 57 & 59 & 55 & 59 & 49 \\
\hline All other fresh fruit & KPRR & 276 & 274 & 290 & 304 & VQGS & 318 & 351 & 343 & 358 & 392 & 394 & 389 & 360 \\
\hline Canned fruit & KPRS & 44 & 37 & 38 & 38 & VQGT & 40 & 39 & 40 & 38 & 36 & 39 & 35 & 32 \\
\hline Dried fruit, nuts and fruit and & & & & & & & & & & & & & & \\
\hline nut products & KPRT & 35 & 34 & 30 & 35 & VQGU & 39 & 41 & 40 & 46 & 51 & 53 & 51 & 52 \\
\hline Fruit juices (ml) & KPRU & 277 & 304 & 284 & 303 & VQGX & 327 & 333 & 322 & 280 & 350 & 366 & 340 & 325 \\
\hline Flour & KPRV & 54 & 55 & 56 & 67 & VQGY & 55 & 61 & 52 & 55 & 60 & 54 & 54 & 63 \\
\hline Bread & KPRW & 746 & 742 & 717 & 720 & VQGZ & 769 & 756 & 728 & 695 & 701 & 692 & 677 & 659 \\
\hline Buns, scones and teacakes & KPRX & 43 & 41 & 40 & 43 & $\mathrm{VQHA}$ & 37 & 41 & 44 & 47 & 46 & 45 & 44 & 43 \\
\hline Cakes and pastries & KPRY & 93 & 88 & 87 & 89 & $\mathrm{VQHB}$ & 139 & 122 & 120 & 117 & 122 & 120 & 115 & 111 \\
\hline Biscuits & KPRZ & 138 & 137 & 132 & 141 & VQHC & 166 & 174 & 163 & 165 & 165 & 165 & 163 & 170 \\
\hline Breakfast cereals & KPSA & 135 & 136 & 134 & 143 & $\mathrm{VQHE}$ & 133 & 132 & 134 & 131 & 135 & 135 & 130 & 130 \\
\hline Oatmeal and oat products & KPSB & 16 & 11 & 13 & 15 & VQHF & 12 & 13 & 12 & 14 & 19 & 17 & 19 & 20 \\
\hline Other cereals and cereal products & JZCG & 293 & 270 & 284 & 291 & VQHG & 345 & 366 & 360 & 354 & 378 & 378 & 387 & 375 \\
\hline Tea & KPSC & 36 & 35 & 32 & 34 & VQHK & 34 & 34 & 31 & 31 & 33 & 30 & 30 & 30 \\
\hline Instant coffee & KPSD & 11 & 12 & 11 & 11 & VQHL & 13 & 12 & 13 & 13 & 13 & 14 & 13 & 14 \\
\hline Canned soups & KPSE & 70 & 71 & 67 & 71 & $\mathrm{VQHM}$ & 79 & 80 & 77 & 76 & 82 & 79 & 79 & 76 \\
\hline Pickles and sauces & KPSF & 92 & 96 & 91 & 107 & $\mathrm{VQHN}$ & 121 & 123 & 121 & 120 & 125 & 128 & 129 & 130 \\
\hline
\end{tabular}

1 See chapter text.

Sources: Living Costs and Food Survey;

2 Including also school and welfare milk (pre-2001-02). 